\title{
Involvement of phosphatase and tensin homolog deleted from chromosome 10 in rodent model of neuropathic pain
}

Shi-Ying Huang ${ }^{1,2+}$, Chun-Sung Sung ${ }^{3,4+}$, Wu-Fu Chen ${ }^{1,5,6}$, Chun-Hong Chen ${ }^{1,7}$, Chien-Wei Feng ${ }^{1,7}$, San-Nan Yang ${ }^{8}$, Han-Chun Hung ${ }^{1,7}$, Nan-Fu Chen 1,9, Pey-Ru Lin ${ }^{10}$, San-Cher Chen ${ }^{2,10}$, Hui-Min David Wang ${ }^{1,11,12,13}$, Tian-Huei Chu ${ }^{10}$, Ming-Hong Tai ${ }^{2,10,14^{*}}$ and Zhi-Hong Wen ${ }^{1,7,15^{*}}$

\begin{abstract}
Background: Many cancer research studies have extensively examined the phosphatase and tensin homolog deleted from chromosome 10 (PTEN) pathway. There are only few reports that suggest that PTEN might affect pain; however, there is still a lack of evidence to show the role of PTEN for modulating pain. Here, we report a role for PTEN in a rodent model of neuropathic pain.

Results: We found that chronic constriction injury (CCl) surgery in rats could elicit downregulation of spinal PTEN as well as upregulation of phosphorylated PTEN (phospho-PTEN) and phosphorylated mammalian target of rapamycin (phospho-mTOR). After examining such changes in endogenous PTEN in neuropathic rats, we explored the effects of modulating the spinal PTEN pathway on nociceptive behaviors. The normal rats exhibited mechanical allodynia after intrathecal (i.t.) injection of adenovirus-mediated PTEN antisense oligonucleotide (Ad-antisense PTEN). These data indicate the importance of downregulation of spinal PTEN for nociception. Moreover, upregulation of spinal PTEN by i.t. adenovirus-mediated PTEN (Ad-PTEN) significantly prevented CCl-induced development of nociceptive sensitization, thermal hyperalgesia, mechanical allodynia, cold allodynia, and weight-bearing deficits in neuropathic rats. Furthermore, upregulation of spinal PTEN by i.t. Ad-PTEN significantly attenuated CCl-induced microglia and astrocyte activation, upregulation of tumor necrosis factor-a (TNF-a) and phospho-mTOR, and downregulation of PTEN in neuropathic rats 14 days post injury.
\end{abstract}

Conclusions: These findings demonstrate that PTEN plays a key, beneficial role in a rodent model of neuropathic pain.

Keywords: Chronic constriction injury, Intrathecal, Astrocyte, Neuroinflammation

\section{Background}

Pain affects 1.5 billion people globally, including 116 million people in the USA and 164 million people in Europe and Israel combined [1]. The 2009 global pain market was estimated to be over US $\$ 50$ billion [2]. Previous studies indicated that chronic pain occurs in about $20 \%$ of the general population $[3,4]$, and the prevalence of neuropathic pain is $6.9 \%$ [4]. In a review of 174 trials

\footnotetext{
* Correspondence: minghongtai@gmail.com; wzh@mail.nsysu.edu.tw

${ }^{\dagger}$ Equal contributors

${ }^{2}$ Center for Neuroscience, National Sun Yat-sen University, No. 70, Lienhai Road, Kaohsiung 80424, Taiwan

'Department of Marine Biotechnology and Resources, National Sun Yat-sen University, No. 70, Lienhai Road, Kaohsiung 80424, Taiwan

Full list of author information is available at the end of the article
}

published, Finnerup et al. [5] reported that there are no drug treatments available that can relieve all neuropathic pain conditions. Moreover, the detailed mechanisms underlying neuropathic pain still remain unclear.

The phosphatase and tensin homolog deleted from chromosome 10 (PTEN) is a tumor suppressor of phosphatased activity [6]. PTEN has been studied extensively through cancer research $[7,8]$; however, there are only few reports that suggest that PTEN might affect pain [9]. Goebbels et al. have demonstrated that by targeted disruption of Pten in Schwann cells that causes focal hypermyelination in the PNS and is associated with progressive peripheral neuropathy in mice [9]. However, there is still a lack of evidence to show the role of PTEN 
for modulating pain. PTEN is considered an upstream inhibitory mediator of mammalian target of rapamycin (mTOR) [10-12]. Several studies have demonstrated that inhibition of the spinal mTOR pathway can attenuate nociception in neuropathic pain $[13,14]$. We suspect that PTEN upregulation may have a therapeutic effect on neuropathic pain.

Here, we use a rat model of chronic constriction injury (CCI)-induced neuropathic pain combined with an intrathecal (i.t.) delivery system to determine whether central PTEN plays a role in neuropathic pain. We examined changes in endogenous spinal PTEN in neuropathic rats using our adenovirus-mediated target gene overexpression system. This system has been shown to be stable and effective in rodent models, especially adenovirusmediated PTEN (Ad-PTEN) in in vitro mouse models [15] and in both ex vivo [16] and in vivo [17] rat models. Additionally, we examined whether modulating the spinal PTEN pathway affected nociceptive behaviors that were measured reliably with behavioral pain assays $[18,19]$. Spinal neuroinflammation may accelerate central sensitization and promote the development and maintenance of neuropathic pain [20,21]. Furthermore, spinal neuroinflammation in CCI is characterized by microglial and astrocytic activation and increased expression of the proinflammatory mediator tumor necrosis factor- $\alpha$ (TNF- $\alpha$ ) $[19,22]$. Many studies have further demonstrated that inhibiting microglial and astrocytic activation can have analgesic effects $[19,23-25]$. Similarly, TNF- $\alpha$ reportedly plays key roles in neuropathic pain [26,27], whereas, inhibition of spinal TNF- $\alpha$ inhibits neuropathic pain behavior [28]. In the present study, we examine whether PTEN affects spinal microglial and astrocytic activation and upregulation of TNF- $\alpha$ accompanied the nociceptive behaviors in CCI rats. In addition, we examine whether PTEN affects downstream signaling of mTOR at the spinal level and in the neuropathic state.

\section{Methods}

\section{Animals}

We housed the male Wistar rats (260 to $285 \mathrm{~g}$ ) for free access to food and water in a temperature-controlled $\left(22^{\circ} \mathrm{C} \pm 1^{\circ} \mathrm{C}\right)$ and light-cycle-controlled (12-h light/12-h dark) room. After the approval by the National Sun Yatsen University and Use Committee, we conformed to the Guiding Principles in the Care and Use of Animals of the American Physiology Society to use rats throughout the experiments. For surgery and drug injections, all rats were anesthetized under isoflurane inhalation (2\%). Then, for preventing infection during the surgery, all rats received intramuscularly postoperative injection of veterin (cefazolin; $0.17 \mathrm{~g} / \mathrm{kg}$ ). Our every effort in experimental design and execution was on the purpose of minimizing the suffering and number of rats we used.

\section{Induction of peripheral mononeuropathy $(\mathrm{CCI})$}

As described by Bennett and Xie [29] and our previous studies $[19,23]$, we performed the surgery of CCI to the right sciatic nerve of rats, to expose the right sciatic nerve of rats (at mid-thigh level), to dissect a 5-mm-long nerve segment of the sciatic nerve, to place four loose ligatures ( 4 to 0 chromic gut) around the sciatic nerve (with 1-mm intervals), and then to suture muscle and skin incision layer by layer. For the sham-operated rats, the surgery was performed only to expose the right sciatic nerve but without ligation.

\section{Implantation of i.t. catheters}

Using the method described in Yaksh and Rudy [30] and our previous studies [19,23], we inserted i.t. catheter (PE5 tubes: 9-cm long, 0.008-in. inner diameter, 0.014in. outer diameter; Spectranetics, Colorado Springs, CO, USA) to the lumbar enlargement of the spinal cord via the atlanto-occipital membrane at the base of the rat's skull down, and then externalized and fixed one end of the i.t. catheter to the cranial aspect of the rat's head for spinal drug administration. Because the dead volume of i.t. catheter was $3.5 \mu \mathrm{L}$, to ensure complete drug delivery, an i.t. artificial cerebrospinal fluid (aCSF) flush $(10 \mu \mathrm{L})$ followed all i.t. injections. The composition of aCSF is as follows: $151.1 \mathrm{mM} \mathrm{Na}^{+}, 2.6 \mathrm{mM} \mathrm{K} \mathrm{K}^{+}, 1.3 \mathrm{mM}$ $\mathrm{Ca}^{2+}, 0.9 \mathrm{mM} \mathrm{Mg}{ }^{2+}, 122.7 \mathrm{mM} \mathrm{Cl}{ }^{-}, 21.0 \mathrm{mM} \mathrm{HCO}_{3}^{-}$, $2.5 \mathrm{mM} \mathrm{HPO}_{4}^{2-}$, and $3.5 \mathrm{mM}$ dextrose and bubbled with $5 \% \mathrm{CO}_{2}$ in $95 \% \mathrm{O}_{2}$ for adjusting the final $\mathrm{pH}$ to 7.3 . Five days after implantation of i.t. catheters, we excluded rats with i.t. catheter that had the fresh blood in the CSF or exhibit of gross neurological injury from the following experiments. According to the method described in other previous study [31] and our previous studies $[19,23]$, we evaluated the locomotor function of rats using the Basso, Beattie, and Bresnahan (BBB) locomotor scale [32].

\section{Preparation of adenovirus vectors}

Using the method described in our previous reports [15-17], we prepared E1- and E3-defective recombinant adenovirus vectors encoding green fluorescent protein (Ad-GFP), PTEN antisense oligonucleotide (Ad-antisense PTEN), or human PTEN cDNA (Ad-PTEN). These adenovirus solutions were tittered with a plaque-forming assay, aliquoted, and then stored at $-80^{\circ} \mathrm{C}$ before their use.

\section{Nociceptive behavioral testing}

(i.) Thermal hyperalgesia test: After we placed each rat into each compartment of clear plastic cages onto an elevated glass platform, we then used an IITC analgesiometer (IITC Inc., Woodland Hills, CA, USA) to test thermal hyperalgesia as described previously by Hargreaves et al. [33] and our previous study [18,34] to position a radiant 
heat source with low-intensity heat (active intensity $=25$ ) onto the middle of the plantar surface of the rat, with a cutoff time of $30 \mathrm{~s}$, to measure the paw withdrawal latency (PWL; in seconds) of the rat until the rat showed a positive sign of pain behavior (licking or withdrawal).

(ii.) Mechanical allodynia test: After we set each rat into each compartment of clear plastic cages onto an elevated metal mesh floor for easy access to the rats' paws, we measured hindpaw withdrawal threshold (PWT; in g) to assess mechanical allodynia using calibrated von Frey filaments (Stoelting, Wood Dale, IL, USA). Then, we apply a series of von Frey filaments of logarithmically incremental stiffness to the midplantar region of the rat hindpaw by Chaplan's 'up-down' method to determine the closest filament to the threshold of pain response (licking or withdrawal) of the rat as described previously by Chaplan et al. [35] and our previous studies [18,19].

(iii.) Cold allodynia test: As described previously by our previous study [18], after we placed each rat in the individual plastic compartments on an elevated metal mesh floor, cold allodynia response (acetone response score; in point) of the rat was monitored during $1 \mathrm{~min}$ following acetone stimulus $(25 \mu \mathrm{l})$ onto the center of the plantar surface of rats' hindpaw. Modified from 4-point scale of previous studies [36,37], acetone response scores of the paw were graded according to a 6-point scale: 0, repeated flicking with persistent licking in $2 \mathrm{~s}$ following acetone stimulus; 1 , prolonged withdrawal or repeated flicking in $2 \mathrm{~s}$ following acetone stimulus; 2, quick and more violent withdrawal, flick, or stamp in $2 \mathrm{~s}$ following acetone stimulus; 3, quick withdrawal, flick, or stamp in $2 \mathrm{~s}$ following acetone stimulus; 4 , withdrawal, flick, or stamp more than 2 s following acetone stimulus; and 5, no response. Then, to sum the four individual scores for obtaining acetone response score of the paw of each rat. The minimum possible total score of the paw could be 0 point (the rat exhibits repeated flicking and licking of paws on each of the four trials), and the maximum possible total score of the paw could be 20 points (the rat exhibits no response to any of the four trials).

(iv.) Weight-bearing test: To let the rat place its hindpaws onto the two force transducers of the incapacitance tester (Singa Technology Corporation, Taoyuan, Taiwan, Republic of China) for measuring the hindpaw weightbearing deficits (change in hindpaw weight distribution; in g) as described in our previous study $[18,38]$. Under normal conditions, the naïve rat distributes weight equally between both hind limbs, but after inducing inflammation of one hind limb (like CCI), the rat redistributes weight for lowering weight-bearing of the affected limb [39]. Change in hindpaw weight distribution of the rat was expressed as a difference by subtracting the affected limb (the right hind limb; the ipsilateral side) from the normal limb (the left hind limb; the contralateral side) measured at the same time.

\section{Spinal immunohistofluorescence analysis}

For reducing variations in immunohistochemical procedures, we mounted the lumbar spinal tissues from the different groups of rats into the same OCT block, sectioned these spinal tissues together using a cryostat at $-30^{\circ} \mathrm{C}$ (HM550; Microm, Waldorf, Germany) and performed the following spinal immunohistofluorescence analysis, with a modified method described in Sung et al. [40] and our previous studies $[18,23]$. The spinal sections $(10 \mu \mathrm{m})$ were incubated with primary antibody, anti-phosphorylated PTEN (anti-phospho-PTEN; Ser380) (1:200 dilution, cat. 9551; Cell Signaling Technology Inc., Beverly, MA, USA; polyclonal rabbit antibody), anti-PTEN (1:200 dilution, cat. 10005059; Cayman Chemical, Ann Arbor, MI, USA; polyclonal rabbit antibody), anti-phosphorylated mTOR (anti-phospho-mTOR; Ser2448) (1:200 dilution, cat. 2976; Cell Signaling Technology Inc., Beverly, MA, USA; polyclonal rabbit antibody), anti-OX-42 (CD11b, microglial marker, 1:200 dilution, cat. CBL1512; EMD Millipore, Temecula, CA, USA; monoclonal mouse antibody), anti-glial fibrillary acidic protein (GFAP) (astrocytic marker, 1:200 dilution, cat. MAB3402; EMD Millipore, Temecula, CA, USA; monoclonal mouse antibody), or anti-TNF- $\alpha$ (1:200 dilution, cat. ARC3012; Life Technologies Corporation, Grand Island, NY, USA) overnight at $4^{\circ} \mathrm{C}$. This was then followed by Alexa Fluor 488-labeled chicken anti-mouse IgG antibody (1:400 dilution, cat. A-21200; Molecular Probes, Eugene, OR, USA; green fluorescence) or DyLight 549-conjugated donkey anti-rabbit IgG antibody (1:400 dilution, cat. 711-506-152; Jackson ImmunoResearch Laboratories Inc., West Grove, PA, USA; red fluorescence) for $40 \mathrm{~min}$ at room temperature. For immunostaining analysis, we use a Leica DM-6000 CS fluorescence microscope (Leica Instruments Inc., Wetzlar, Germany) for examination of these stained spinal sections, and then use a SPOT Xplorer Digital camera (Diagnostic Instruments, Inc., Sterling Heights, MI, USA) for photographing all immunofluorescence images of phospho-PTEN, PTEN, phospho-mTOR, OX-42, GFAP, and TNF- $\alpha$, respectively. We measured the pixel values of the immunoreactivepositive area (using three sections per rat) by Image J software (National Institutes of Health, Bethesda, MD, USA). Spinal neurons located in the superficial laminae, laminae I to III, respond to nociceptive stimuli and directly participate in the transmission of nociception to the brain [41], and thereby the superficial laminae play a more important role in neuropathic pain than the deep laminae. Therefore, in accordance to the method used for neuropathic rodents [41-44], we focused on quantifying the immunoreactivity of the targeted protein in the superficial laminae of the spinal cord. The immunofluorescence data were expressed as a percentage change compared to shamoperated or sham-operated plus i.t. vehicle group, which were considered to be $100 \%$. For double-immunofluorescent 
staining of PTEN and neuronal marker, the spinal sections were incubated with a mixture of anti-PTEN (1:200 dilution) and anti-Neuronal Nuclei (NeuN) (neuronal-specific nuclear protein; neuronal marker, 1:500, Alexa Fluor 488 conjugated antibody, cat. MAB377X, EMD Millipore, Temecula, CA, USA; monoclonal mouse antibody) antibodies overnight at $4^{\circ} \mathrm{C}$, and then followed by DyLight 549-conjugated anti-rabbit IgG antibody (1:400 dilution) for $40 \mathrm{~min}$ at room temperature. On the other hand, for double-immunofluorescent staining of PTEN and microglial marker or astrocytic marker, the spinal sections were incubated with a mixture of anti-PTEN (1:200 dilution) and OX-42 (1:200 dilution) or GFAP (1:200 dilution) antibodies overnight at $4{ }^{\circ} \mathrm{C}$, and then followed by a mixture of Alexa Fluor 488-conjugated anti-mouse IgG antibody (1:400 dilution) and DyLight 549-conjugated anti-rabbit IgG antibody (1:400 dilution) for $40 \mathrm{~min}$ at room temperature. For double-immunofluorescent staining of anti-TNF- $\alpha$ or phospho-mTOR and astrocytic marker, the spinal sections were incubated with a mixture of anti-TNF- $\alpha$ (1:200 dilution) or anti-phospho-mTOR (1:200 dilution) and GFAP (1:200 dilution) antibodies overnight at $4^{\circ} \mathrm{C}$, and then followed by a mixture of DyLight 549-conjugated antirabbit IgG antibody (1:400 dilution) and Alexa Fluor 633conjugated goat anti-mouse IgG antibody (1:400 dilution, cat. A21052; Life Technologies Corporation) for $40 \mathrm{~min}$ at room temperature. The double-immunostaining images were examined and acquired with Leica TCS SP5 II confocal microscope (Leica Instruments Inc., Wetzlar, Germany). We set the color of TNF- $\alpha$ or phospho-mTOR for $549-\mathrm{nm}$ excitation line as pseudo red and set the color of GFAP for $633 \mathrm{~nm}$ excitation line as pseudo green. When co-localization of two proteins occurred, the merge of above two colors, pseudo red (TNF- $\alpha$ or phosphomTOR) and pseudo green (GFAP), yielded yellow color.

\section{Western blot analysis}

Following our published method [22,34,45], we performed Western blotting analysis on the ipsilateral side of the lumbar spinal dorsal samples from the rats. For Western blotting analysis, we collected and washed spinal dorsal samples from rats with ice-cold PBS, and then used a Polytron homogenizer (5 cycles of $10 \mathrm{~s}$ at $3,000 \mathrm{rpm})$ to homogenize in ice-cold lysis buffer $(1 \mu \mathrm{g} / \mathrm{ml}$ aprotinin, $50 \mathrm{mM}$ Tris, $150 \mathrm{mM} \mathrm{NaCl}, 100 \mu \mathrm{g} / \mathrm{ml}$ phenylmethylsulfonyl fluoride, $1 \%$ Triton X-100, $\mathrm{pH} 7.5$ ). After centrifuging at $20,000 \times g$ for $60 \mathrm{~min}$ at $4^{\circ} \mathrm{C}$, we retained this supernatant for Western blot analysis of phosphoPTEN and PTEN. Modified from the method of Lowry et al. [46], we determined the protein concentrations of the supernatant with the DC protein assay kit (Bio-Rad, Hercules, CA, USA). We added an equal volume of sample buffer (2\% 2-mercaptoethanol, 10\% glycerol, 0.1\% bromophenol blue, $50 \mathrm{mM}$ Tris- $\mathrm{HCl}, \mathrm{pH} 7.2$, and $2 \%$ sodium dodecyl sulfate (SDS)) to the supernatant. Then, we electrophoresed the proteins of supernatant through a tricine SDS-polyacrylamide gel at $150 \mathrm{~V}$ for $90 \mathrm{~min}$ and transferred the proteins within the gel to a polyvinylidene difluoride membrane (PVDF membrane; Immobilon-P, Millipore, $0.45-\mu \mathrm{M}$ pore size) in transfer buffer $(380 \mathrm{mM}$ glycine, $1 \%$ SDS, $50 \mathrm{mM}$ Tris- $\mathrm{HCl}, 20 \%$ methanol) at $125 \mathrm{~mA}$ overnight at $4^{\circ} \mathrm{C}$. With blocking PVDF membrane for $1 \mathrm{~h}$ at room temperature using 5\% non-fat dry milk in Tris-buffered saline (TTBS; $137 \mathrm{mM} \mathrm{NaCl}, 0.1 \%$ Tween 20, $20 \mathrm{mM}$ Tris- $\mathrm{HCl}, \mathrm{pH} 7.4$ ), we then incubated the PVDF membrane with antibodies against phospho-PTEN (Ser380; 1:1,000 dilution) or PTEN (1:1,000 dilution) proteins for $180 \mathrm{~min}$ at room temperature. The antibodies recognized immunoreactive bands of phospho-PTEN (approximately $54 \mathrm{kDa}$ ) and PTEN (approximately $47 \mathrm{kDa}$ ) protein, which were visualized with enhanced chemiluminescence (ECL kit; Millipore) and photographed using the UVP BioChemi imaging system (UVP LLC, Upland, CA, USA), respectively. Finally, we performed the relative densitometric quantification of the immunoreactive bands of phospho-PTEN and PTEN protein with LabWorks 4.0 software (UVP LLC, Upland, CA, USA), and relative variations between the bands of the sham-operated or shamoperated plus i.t. vehicle group and the other groups were calculated using the same image. In addition, we reprobed the PVDF membranes using an anti- $\beta$-actin antibody (1:2,500 dilution; catalog no. A5441; Sigma Co., Ltd., St Louis, MO, USA; monoclonal mouse antibody), which was the loading control.

\section{Data and statistical analysis}

We showed all following data as means \pm standard error on the mean (SEM). For statistical analysis, we calculated differences between groups of rats by a one-way analysis of variance (ANOVA), used the Student-Newman-Keuls post hoc test, and then defined the criterion for statistical significance as $P<0.05$.

\section{Results}

The time course of changes of endogenous spinal PTEN in neuropathic rats

To explore any potential changes in the spinal PTEN pathway in neuropathic rats, we prepared the lumbar spinal tissues from the following eight groups of rats: (1) 1 day after sham operation, (2) 3 days after sham operation, (3) 7 days after sham operation, (4) 14 days after sham operation, (5) 1 day after CCI, (6) 3 days after CCI, (7) 7 days after CCI, and (8) 14 days after CCI. We performed a spinal immunohistofluorescence analysis using three antibodies against phospho-PTEN, PTEN, and phospho-mTOR to evaluate the expression of the PTEN pathway in the ipsilateral side of the lumbar spinal dorsal gray matter in neuropathic rats. Compared with the 
sham-operated group (Figure 1A,D), immunoreactivity increased for phospho-PTEN but decreased for PTEN, 7 (Figure 1B,E) and 14 days (Figure 1C,F) after CCI. Similar to the findings in a previous study [47], phospho-mTOR immunoreactivity increased 7 (Figure $1 \mathrm{H}$ ) and 14 days (Figure 1I) after CCI, as compared with that of the shamoperated group (Figure 1G). Quantification of the immunoreactivity results further confirmed phospho-PTEN (Figure 1J) and phospho-mTOR (Figure 1L) upregulation, as well as PTEN downregulation (Figure 1K), 3, 7, and 14 days after CCI. Further, we used Western blot analysis to verify changes in endogenous PTEN in the dorsal horn of the lumbar spinal cord (Figure 2A). The Western blot analysis revealed upregulation of phospho-PTEN (Figure 2B) and downregulation of PTEN (Figure 2C) 14 days after $\mathrm{CCI}$ surgery. To examine the cellular specificity of endogenous PTEN in the dorsal horn of the lumbar spinal cord, we prepared lumbar spinal tissues from the sham-operated group for double-immunofluorescent staining. Confocal double-immunostaining images of the lumbar spinal dorsal gray matter of sham-operated rats further confirmed that most PTEN signals were more often co-localized with GFAP-positive cells (astrocytes, Figure 2E) than NeuN-positive (neuronal cells; Figure 2D) or OX-42-positive cells (microglial cells, Figure 2F).

The effect of i.t. Ad-antisense PTEN on nociceptive responses To explore the effects of modulating spinal PTEN pathway on nociceptive behaviors in normal rats, we prepared the following five groups of rats: (1) naïve, (2) i.t. vehicle, (3) i.t. Ad-GFP, (4) i.t. Ad-antisense PTEN, and (5) i.t. Ad-PTEN. Five days after implantation of the i.t. catheters, the rats received i.t. injection of vehicle, AdGFP, Ad-antisense PTEN, or Ad-PTEN. No significant

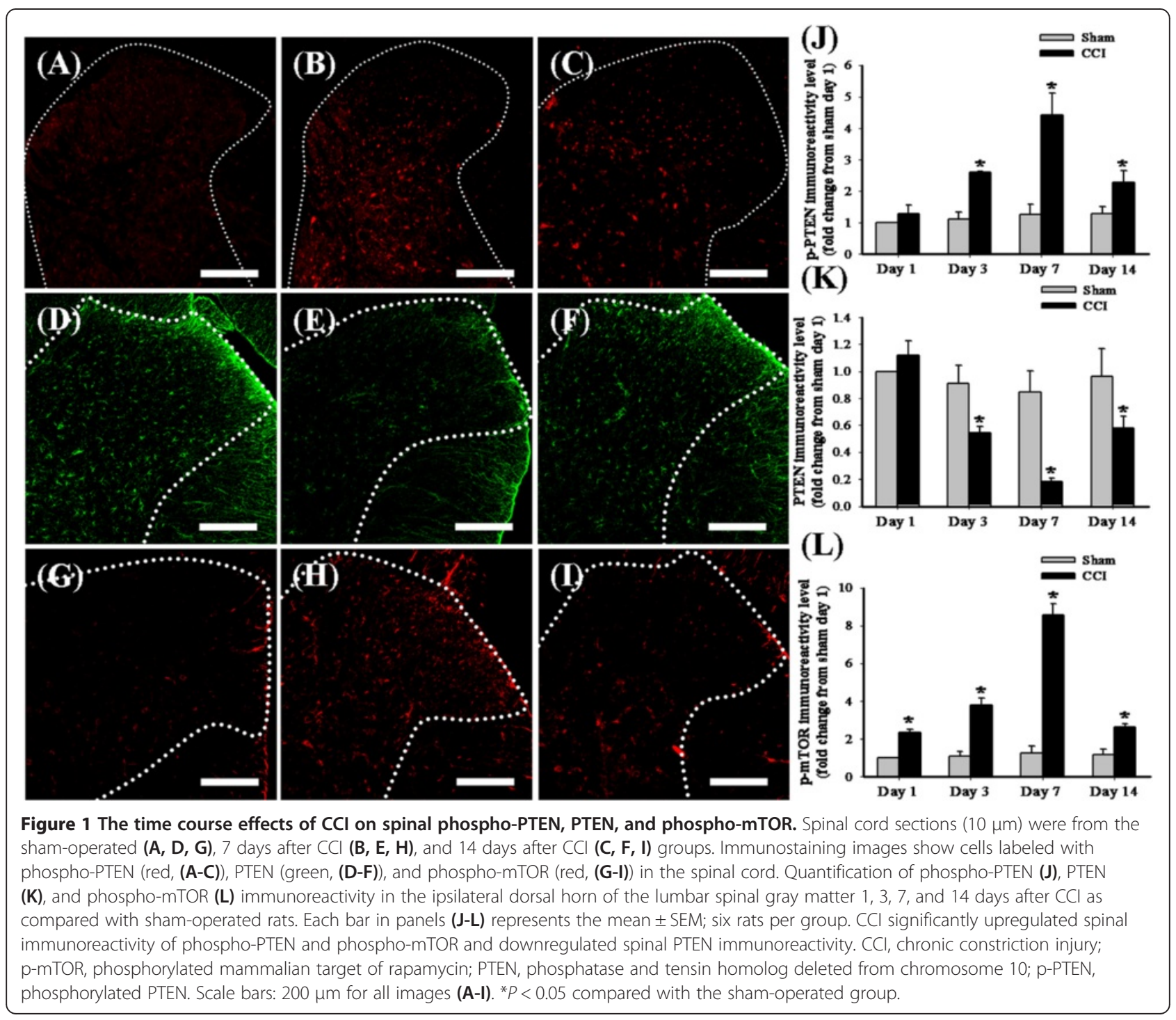




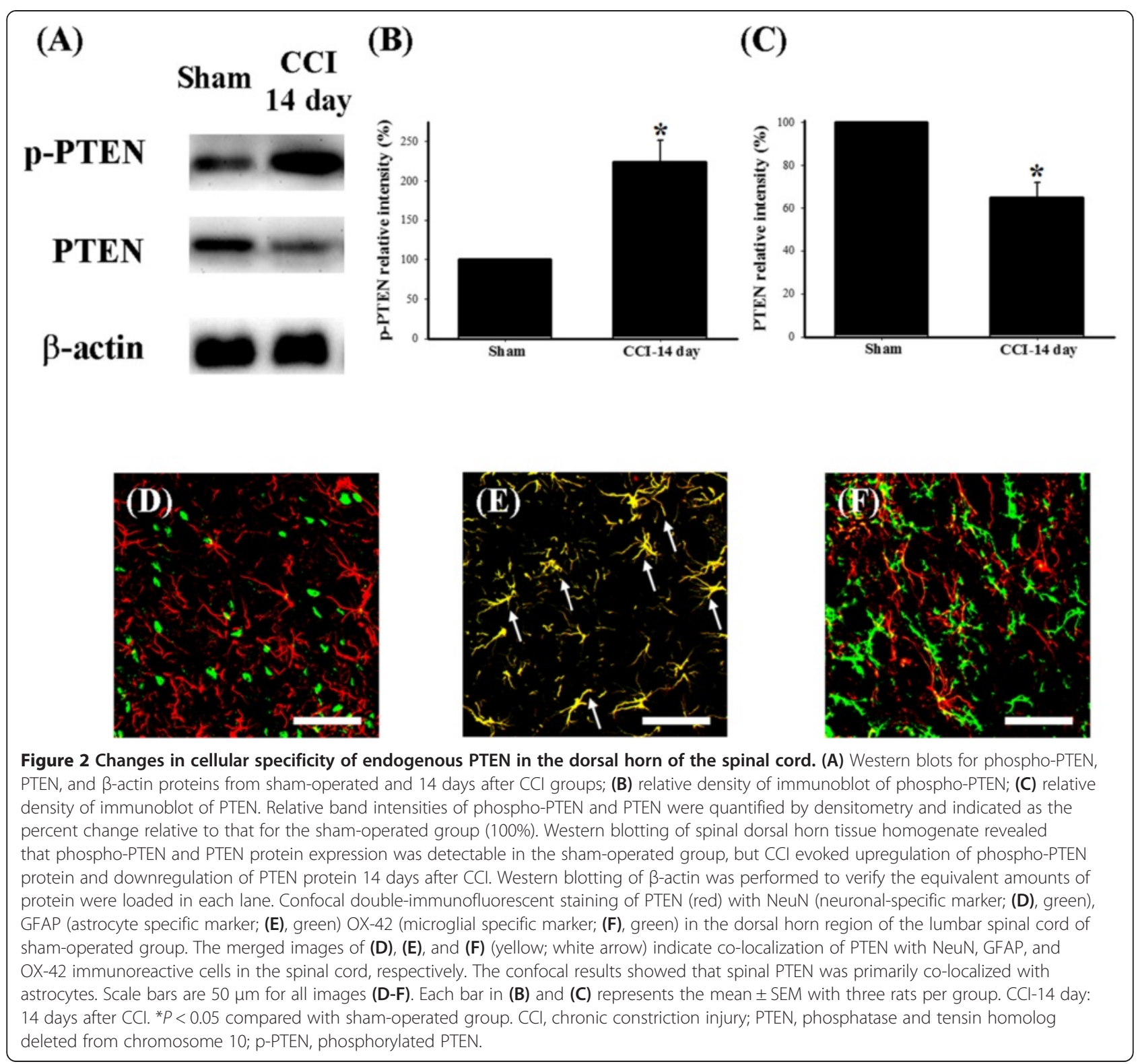

differences were observed between the naïve group (data not shown) and the i.t. vehicle group for PWL, PWT, or acetone response scores. Compared with i.t. vehicle group, i.t. Ad-GFP did not significantly affect PWL (Figure 3A), PWT (Figure 3B), and acetone response score (Figure $3 C$ ) of rats. After i.t. injection of Adantisense PTEN, the rats exhibited mechanical allodynia (Figure $3 \mathrm{~B}$ ) but not thermal hyperalgesia (Figure 3A) and cold allodynia (Figure $3 \mathrm{C}$ ) from 3 to 7 days compared with that of i.t. Ad-GFP group. Compared with i.t. Ad-GFP group, i.t. Ad-PTEN did not significantly affect PWL (Figure 3A), PWT (Figure 3B), and acetone response score (Figure $3 \mathrm{C}$ ) of rats. In addition, in the present study, i.t. Ad-GFP, Ad-antisense PTEN, or Ad-
PTEN-treated rats did not exhibit any obvious abnormal external behavior (including locomotor function).

The inhibitory effects of Ad-PTEN on nociception in neuropathic rats

To verify that Ad-PTEN has the ability to upregulate production of spinal PTEN, we prepared the following three groups of rats: (1) naïve plus i.t. vehicle, (2) naïve plus i.t. Ad-GFP, and (3) naïve plus i.t. Ad-PTEN. We observed no significant difference in spinal PTEN expression between naïve rats plus i.t. vehicle (Figure 4A) and rats examined 14 days after i.t. injection of Ad-GFP (Figure 4B), whereas PTEN immunoreactivity increased 14 days after i.t. Ad-PTEN (Figure 4C). Quantification of 


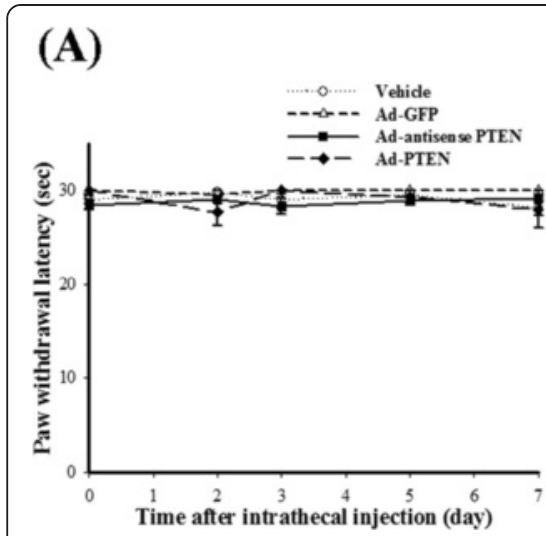

(B)

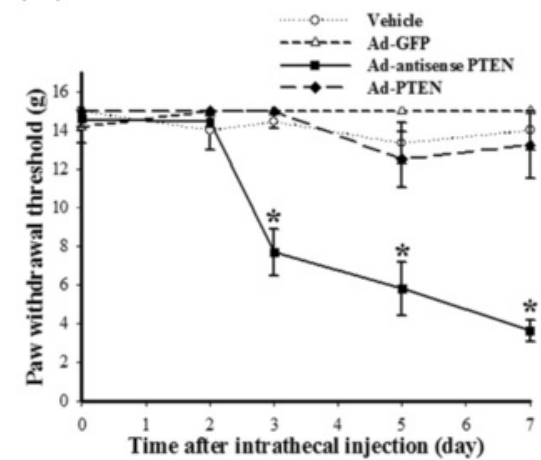

(C)

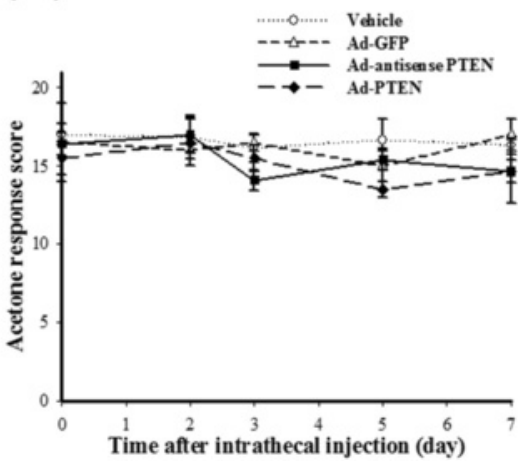

Figure 3 Time course of the effects of i.t. Ad-antisense PTEN on nociceptive behaviors in normal rats. The naive rats exhibited mechanical allodynia (B) but not thermal hyperalgesia (A) and cold allodynia (C) from 3 to 7 days after i.t. injection of Ad-antisense PTEN. These data indicate the importance of downregulation of spinal PTEN for nociception. Each point represents the mean \pm SEM with six rats per group. ${ }^{*} P<0.05$ compared with vehicle group. Ad-GFP, adenovirus-mediated green fluorescent protein; Ad-PTEN, adenovirus-mediated phosphatase and tensin homolog deleted from chromosome 10; Ad-antisense PTEN, adenovirus-mediated PTEN antisense oligonucleotide.

the immunoreactivity results (Figure 4D) further confirmed that PTEN upregulation in the naïve plus i.t. Ad-PTEN group 14 days after i.t. injection. Confocal double-immunostaining images of the lumbar spinal dorsal gray matter further confirmed most PTEN signals were more often co-localized with GFAP-positive cells (astrocytes; Figure 4F) than NeuN-positive (neuronal cells; Figure 4E) or OX-42-positive cells (microglial cells; Figure 4G) in the naïve rats administered i.t. Ad-PTEN.

To examine whether spinal PTEN upregulation induced with i.t. Ad-PTEN affected neuropathic pain behaviors, we prepared the following three groups of rats: (1) CCI plus i.t. vehicle, (2) CCI plus i.t. Ad-GFP, and (3) CCI plus i.t. Ad-PTEN. Five days after the i.t. catheters were implanted, the rats underwent CCI surgery, and the vehicle, Ad-GFP, or Ad-PTEN was administered i.t. immediately afterwards. Similar to previous findings [48], CCI promoted the development and maintenance of nociceptive behaviors in rats. Compared with the vehicle group, i.t. Ad-GFP did not significantly affect PWL (Figure 5A), PWT (Figure 5B), acetone response score (Figure 5C), and weight-bearing deficits (Figure 5D) for the ipsilateral hindpaw of CCI rats. Compared with i.t. Ad-GFP group, i.t. Ad-PTEN significantly attenuated CCI-induced thermal hyperalgesia (Figure 5A), mechanical allodynia (Figure 5B), cold allodynia (Figure 5C), and weight-bearing deficits (Figure 5D) up to 14 days post injury. In contrast, similar to previous studies $[49,50]$, the contralateral hindpaw in CCI rats did not show pain-related behavior (Figure 6). Compared with i.t. vehicle group, neither i.t. Ad-GFP nor Ad-PTEN significantly affected PWL (Figure 6A), PWT (Figure 6B), or acetone response scores (Figure $6 \mathrm{C}$ ) for the contralateral hindpaw of CCI rats. In addition, i.t. Ad-GFP- and
Ad-PTEN-treated CCI rats failed to show obvious abnormal behaviors (including locomotor function), and the naïve-plus-i.t. Ad-PTEN rats exhibited normal locomotor function. We then focused on 14 days post injury to determine whether the modulation of spinal neuroinflammatory processes is involved in the maintenance of the antinociceptive effects of the upregulation of spinal PTEN by i.t. Ad-PTEN.

\section{The effect of Ad-PTEN on spinal neuroinflammation in neuropathic rats}

For spinal immunohistofluorescence assay, we collected spinal tissue on day 14 post injury from the following three groups of rats: (1) sham-operated plus i.t. vehicle, (2) CCI plus i.t. Ad-GFP, and (3) CCI plus i.t. Ad-PTEN. OX-42-, GFAP-, and TNF- $\alpha$-immunoreactive cells were scattered throughout the ipsilateral dorsal horn of the lumbar spinal gray matter of sham-operated plus i.t. vehicle (Figure 7A,D,G), CCI plus i.t. Ad-GFP (Figure 7B, E,H), and CCI plus i.t. Ad-PTEN (Figure 7C,F,I) groups. Similarly, and as previously reported,[19,22] the immunoreactivity of OX-42 (Figure 7B), GFAP (Figure 7E), and TNF- $\alpha$ (Figure $7 \mathrm{H}$ ) of CCI plus i.t. Ad-GFP group were upregulated on day 14 post injury when compared with the sham-operated plus i.t. vehicle group. CCI-induced upregulation of OX-42 (Figure 7C), GFAP (Figure 7F), and TNF- $\alpha$ (Figure 7I) were inhibited by i.t. Ad-PTEN. Quantification of OX-42 (Figure 7J), GFAP (Figure 7K), and TNF- $\alpha$ (Figure $7 \mathrm{~L}$ ) immunoreactivity supported the finding that inhibition of CCI-induced upregulation of OX-42 and GFAP, which are microglial and astrocytic immunohistochemical activation markers, as well as TNF- $\alpha$, are consistent with the anti-nociceptive effects of i.t. Ad-PTEN. 

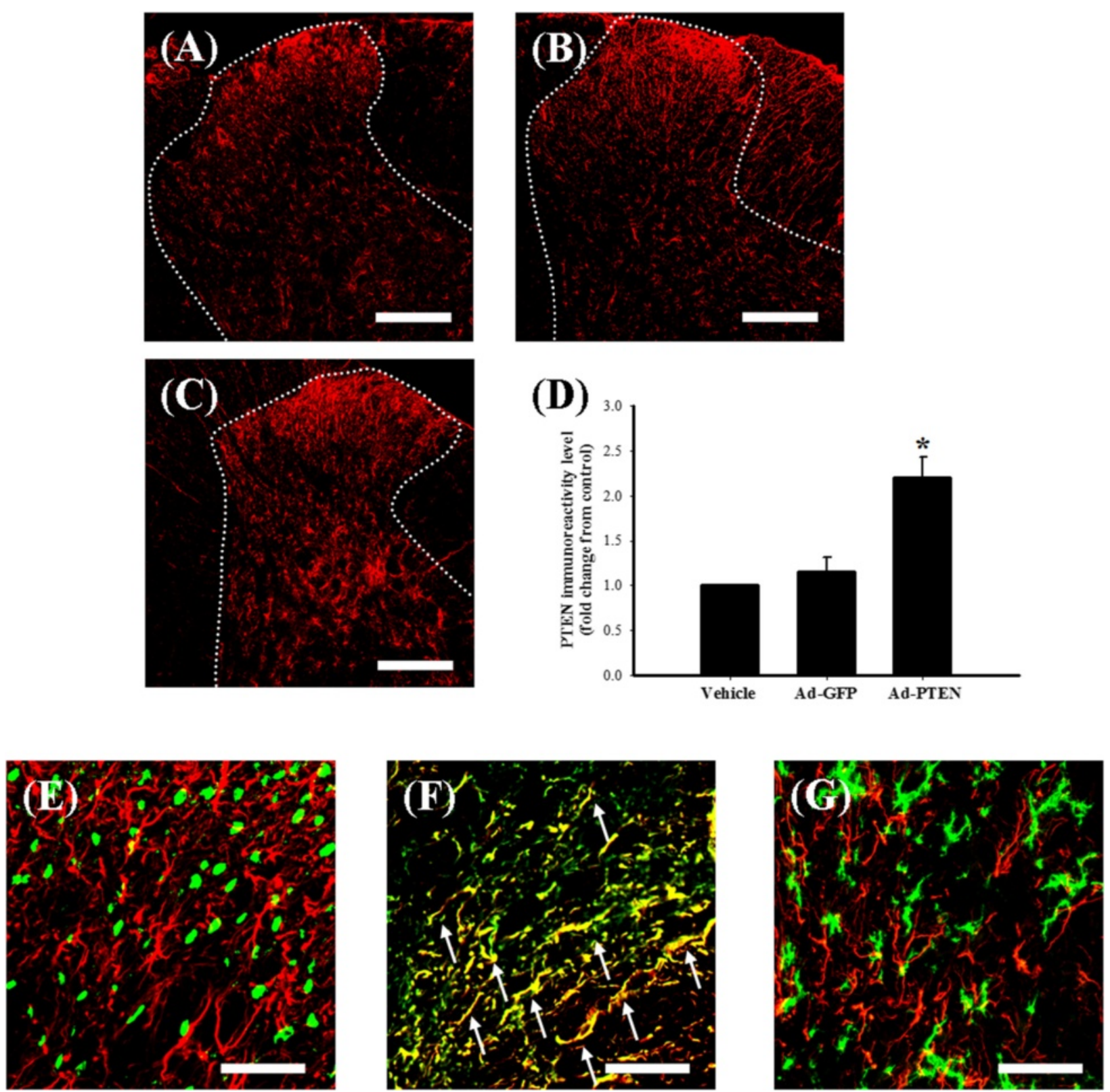

Figure 4 The effects of i.t. injection of Ad-PTEN on PTEN in the dorsal lumbar spinal cord. Fourteen days after i.t.; spinal cord sections $(10 \mu \mathrm{m})$ are from i.t. injection of vehicle (A), Ad-GFP (B), and Ad-PTEN (C) groups. Immunostaining images of the spinal cord show cells labeled with PTEN (red). Quantification of PTEN (D) immunoreactivity in the ipsilateral dorsal horn of the lumbar spinal gray matter compared with vehicle group. Injection (i.t.) of Ad-PTEN significantly upregulated spinal PTEN immunoreactivity. Each bar in (D) represents the mean \pm SEM with six rats per group. Ad-GFP, adenovirus-mediated green fluorescent protein; Ad-PTEN, adenovirus-mediated phosphatase and tensin homolog deleted from chromosome 10. Confocal double-immunofluorescent staining of PTEN (red) with NeuN (neuronal-specific marker; (E), green), GFAP (astrocyte specific marker; (F), green), and OX-42 (microglial specific marker; (G), green) in the dorsal horn region of the lumbar spinal cord of the i.t. Ad-PTEN group. The merged images of (E), (F), and (G) (yellow; white arrow) indicate co-localization of PTEN with NeuN, GFAP, and OX-42 immunoreactive cells in the spinal cord, respectively. The confocal results show that spinal PTEN was primarily co-localized with astrocytes in i.t. Ad-PTEN group. Scale bars are $200 \mu \mathrm{m}$ for panels A-C and $50 \mu \mathrm{m}$ for panels $\mathrm{E}-\mathrm{G}$. ${ }^{*} \mathrm{P}<0.05$ compared with the vehicle group.

The effects of Ad-PTEN on spinal astrocytic PTEN signaling in $\mathrm{CCl}$ rats

We further examined the effects of i.t. Ad-PTEN on CCI-induced changes in the spinal PTEN pathway. We further used Western blot analysis to measure changes in PTEN and phospho-mTOR in the dorsal horn of the lumbar spinal cord from the following six groups of rats: (1) sham-operated plus i.t. vehicle 7 days post injury, (2) 

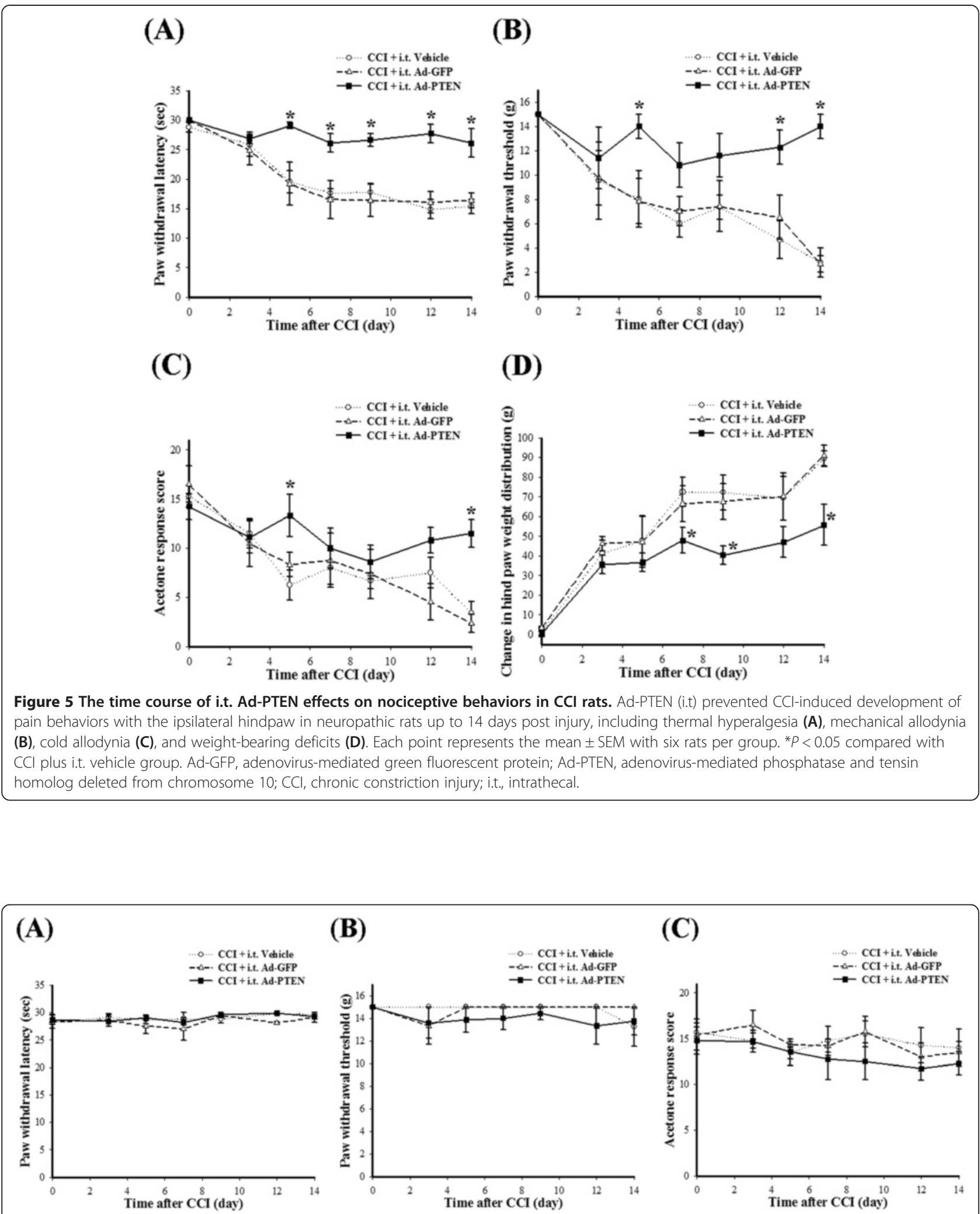

(B)

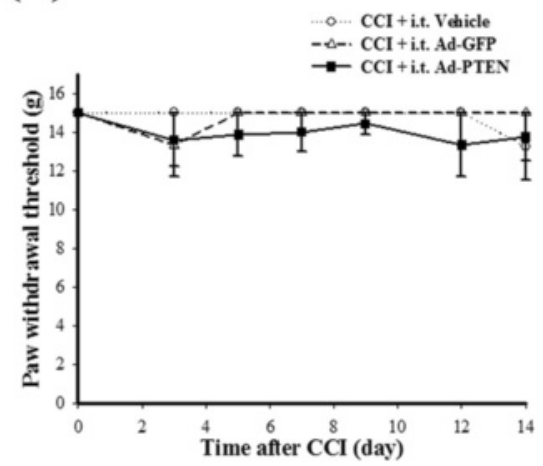

(C)

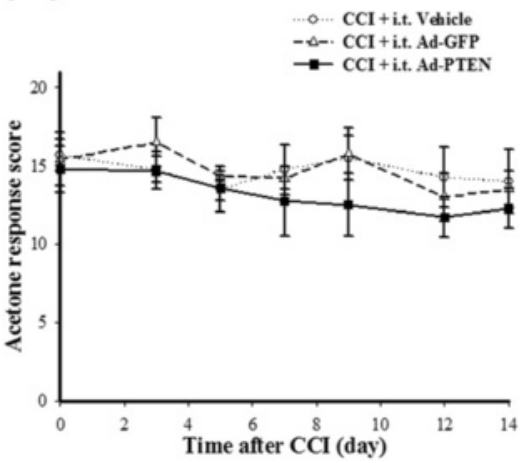

Figure 6 The effects of i.t. Ad-PTEN on nociceptive behaviors with the contralateral hindpaw in CCI rats. I.t. Ad-PTEN did not significantly affect the responses to thermal hyperalgesia (A), mechanical allodynia (B), or cold allodynia (C) tests. Each point represents the mean \pm SEM with six rats per group. Ad-GFP, adenovirus-mediated green fluorescent protein; Ad-PTEN, adenovirus-mediated phosphatase and tensin homolog deleted from chromosome 10; CCl, chronic constriction injury; i.t., intrathecal. 


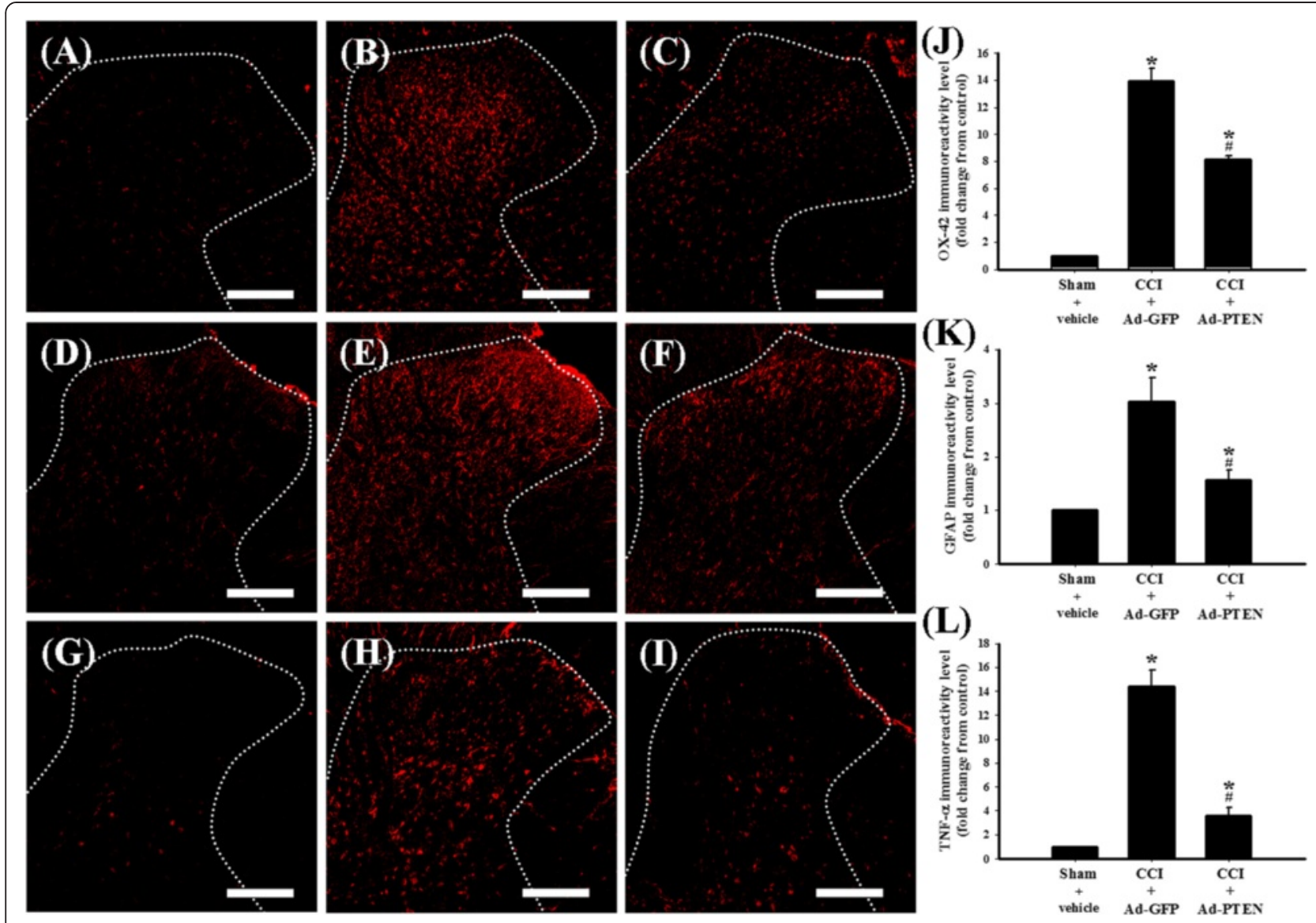

Figure 7 The effects of i.t. Ad-PTEN on CCl-induced microglial and astrocytic activation and upregulation of TNF-a. Spinal cord sections $(10 \mu \mathrm{m})$ obtained 14 days post-surgery from sham-operated plus i.t. vehicle $(\mathbf{A}, \mathbf{D}, \mathbf{G}), \mathrm{CCl}$ plus i.t. Ad-GFP $(\mathbf{B}, \mathbf{E}, \mathbf{H})$, and CCl plus i.t. Ad-PTEN (C, F, I) groups. Immunostaining images show cells labeled with OX-42 (red; (A-C)) and GFAP (red; (D-F)), TNF-a (red; (G-I)) in the spinal cord. Quantification of OX-42 (J) and GFAP (K), and TNF-a (L) immunoreactivity in the ipsilateral dorsal horn of the lumbar spinal gray matter. Ad-GFP, adenovirus-mediated green fluorescent protein; Ad-PTEN, adenovirus-mediated phosphatase and tensin homolog deleted from chromosome 10; CCl, chronic constriction injury; GFAP, glial fibrillary acidic protein; TNF, tumor necrosis factor. Each bar in (J-L) represents the mean \pm SEM with six rats per group. Ad-PTEN (i.t.) significantly inhibited CCl-induced upregulation of spinal OX-42, GFAP, and TNF-a immunoreactivity. Scale bars: $200 \mu \mathrm{m}$ for all images (A-I). ${ }^{*}<0.05$ compared with sham-operated plus i.t. vehicle group; ${ }^{\#} P<0.05$ compared with CCl plus i.t. Ad-GFP.

CCI plus i.t. Ad-GFP 7 days post injury, (3) CCI plus i.t. Ad-PTEN 7 days post injury, (4) sham-operated plus i.t. vehicle 14 days post injury, (5) CCI plus i.t. Ad-GFP 14 days post injury, and (6) CCI plus i.t. Ad-PTEN 14 days post injury. The Western blot analysis revealed both a downregulation of PTEN (Figure 8B) and an upregulation of phospho-mTOR (Figure $8 \mathrm{C}$ ) in the spinal cord on days 7 and 14 after CCI surgery, which were both attenuated by i.t. Ad-PTEN. Compared with the sham-operated plus i.t. vehicle group (Figure 9A), PTEN immunoreactivity in the CCI plus i.t. Ad-GFP group (Figure 9B) decreased 14 days post injury. Compared with the i.t. Ad-GFP group, i.t. Ad-PTEN (Figure 9C) attenuated CCI-induced downregulation of PTEN 14 days post injury. Compared with the sham-operated plus i.t. vehicle group (Figure 9D), phospho-mTOR immunoreactivity in the CCI plus i.t. Ad-GFP group (Figure 9E) increased 14 days post injury. Compared with i.t. Ad-GFP group, i.t. Ad-PTEN (Figure 9F) attenuated CCI-induced upregulation of phospho-mTOR 14 days post injury. Quantification of the immunoreactivity results further confirmed that i.t. Ad-PTEN significantly attenuated CCI-induced downregulation of PTEN (Figure 9G) and upregulation of phospho-mTOR (Figure 9H) 14 days post injury. To examine the inhibitory effects of i.t. injection of AdPTEN on CCI-induced upregulation of TNF- $\alpha$ and phospho-mTOR in spinal astrocytes, we prepared tissue 14 days post-surgery from sham-operated plus i.t. vehicle (Figure 10A,D), CCI plus i.t. Ad-GFP (Figure 10B,E), and CCI plus i.t. Ad-PTEN (Figure 10C,F) rats to perform double-immunofluorescent staining. The confocal doubleimmunostaining results showed that spinal TNF- $\alpha$ (red; 


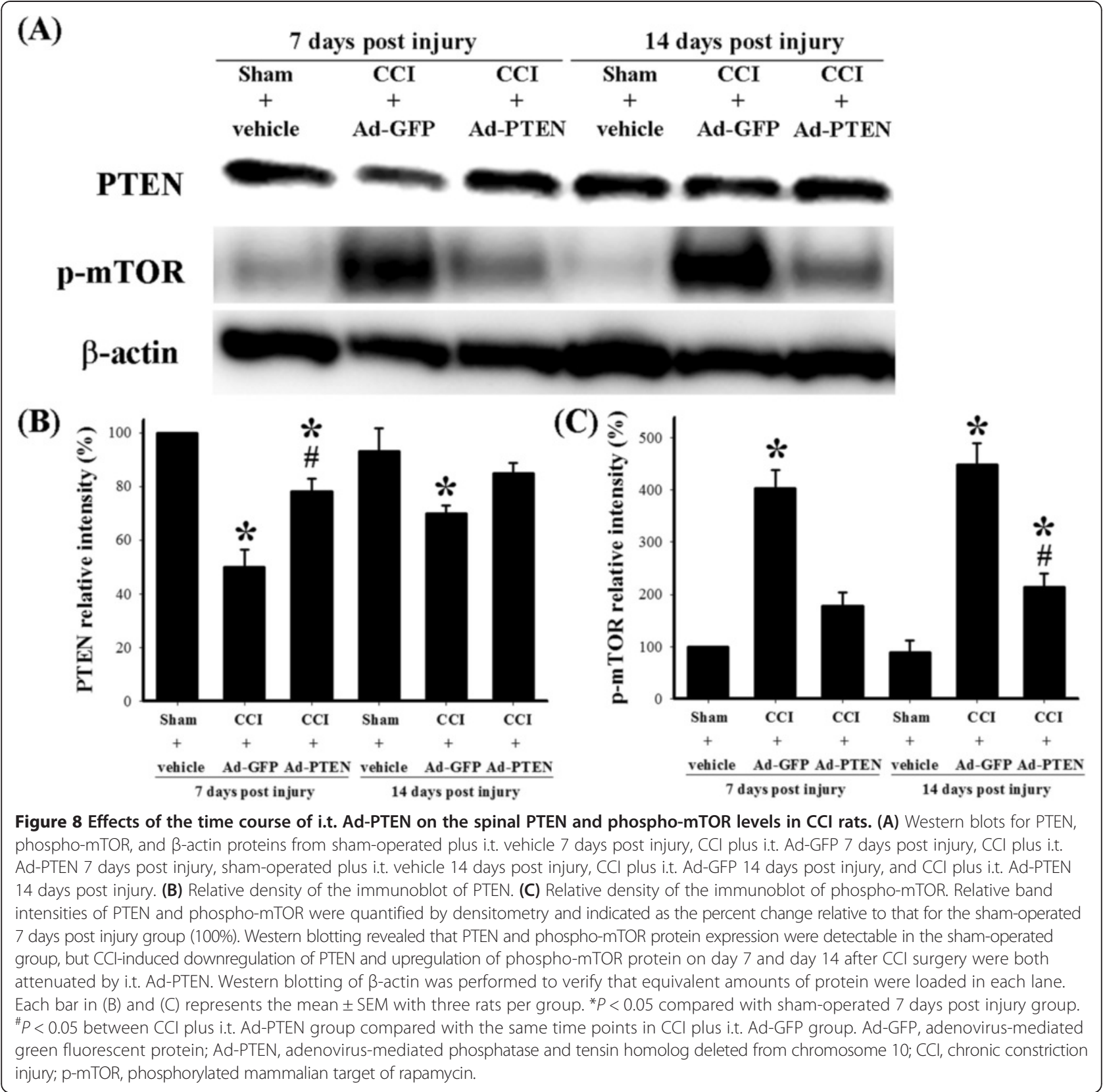

Figure 10B) and phospho-mTOR (red; Figure 10E) were primarily co-localized with astrocytes in the CCI group, and i.t. Ad-PTEN significantly attenuated CCI-induced upregulation of spinal TNF- $\alpha$ (Figure 10C) and phosphomTOR (Figure 10F) immunoreactivity in astrocytes.

\section{Discussion}

The role of endogenous PTEN in neuropathic and normal rats

Previous neuroscience studies have focused on the role of PTEN in axon regeneration [11,51,52], Alzheimer's disease [53], Parkinson's disease [54], ischemic brain injury [12], and spinal cord injury [52,55-57]. To the best of our knowledge, the present report is the first to explore the role of spinal PTEN in pain, especially neuropathic pain. Using a spinal immunohistofluorescence assay system and Western blot analysis, we found that CCI could elicit downregulation of ipsilateral dorsal spinal PTEN in rats (Figures 1 and 2). Phosphorylation of Ser-380 is known to suppress phosphatase activity of PTEN [58]. We also demonstrated that upregulation of phospho-PTEN was associated with downregulation of PTEN 14 days after CCI surgery (Figures 1 and 2). Phosphorylation of Ser-2448 mTOR is a biomarker for 

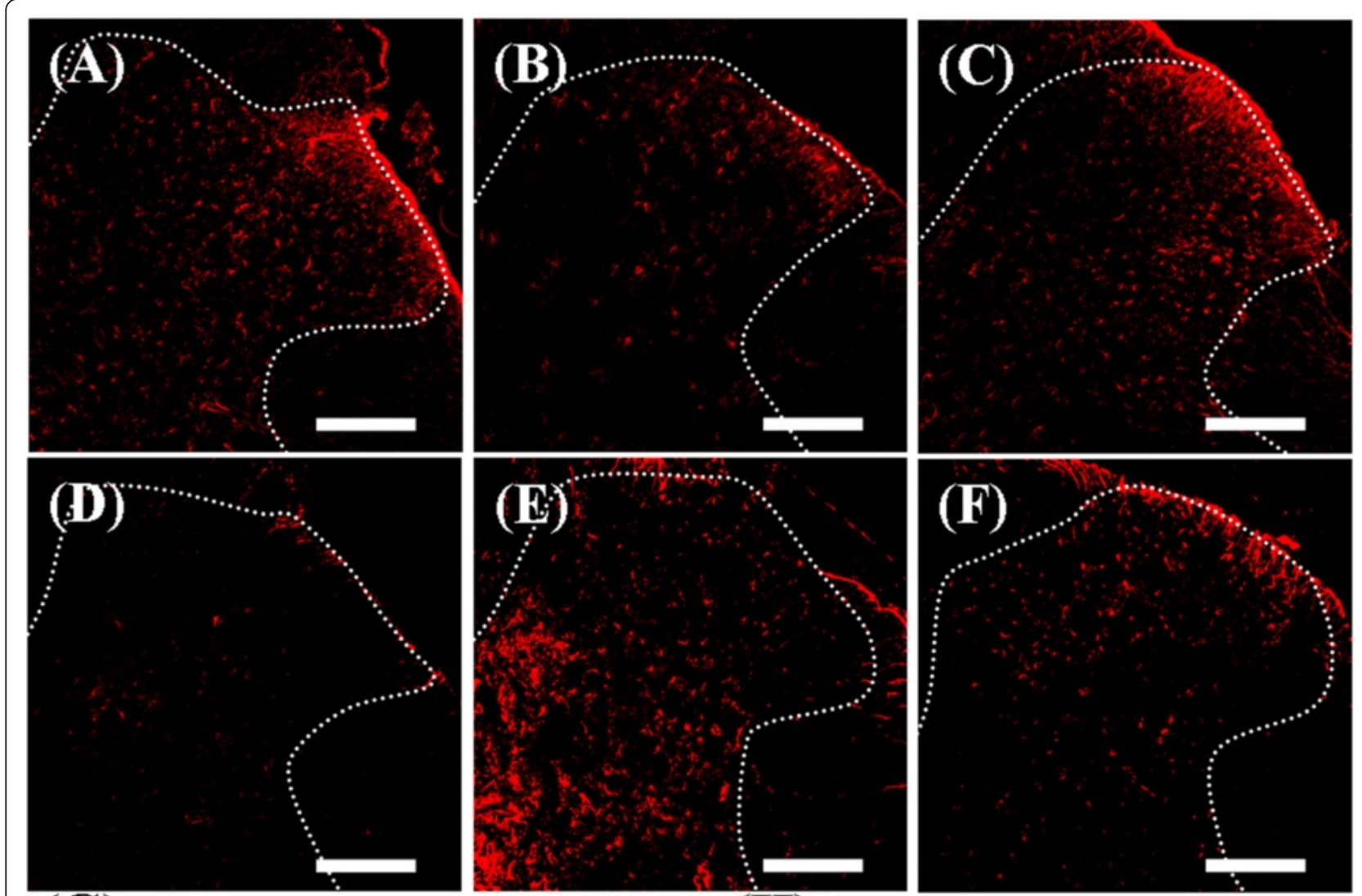
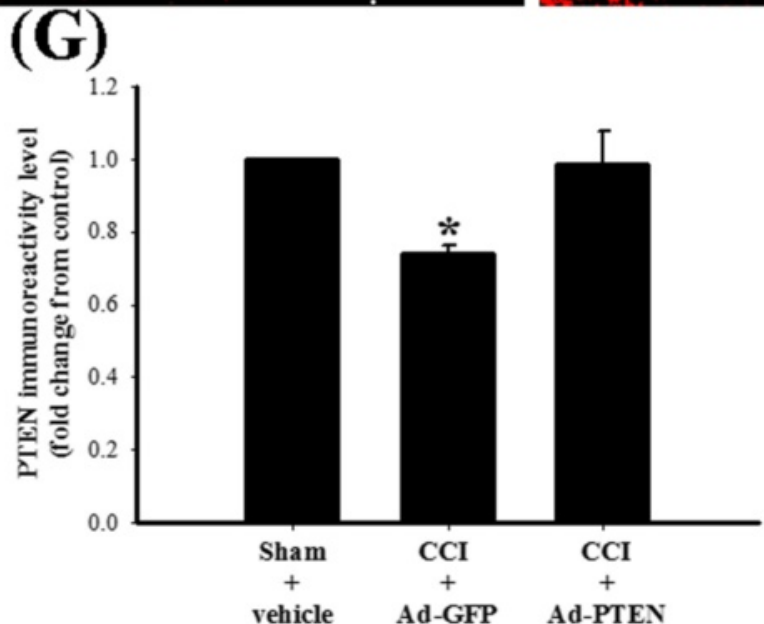

(H)

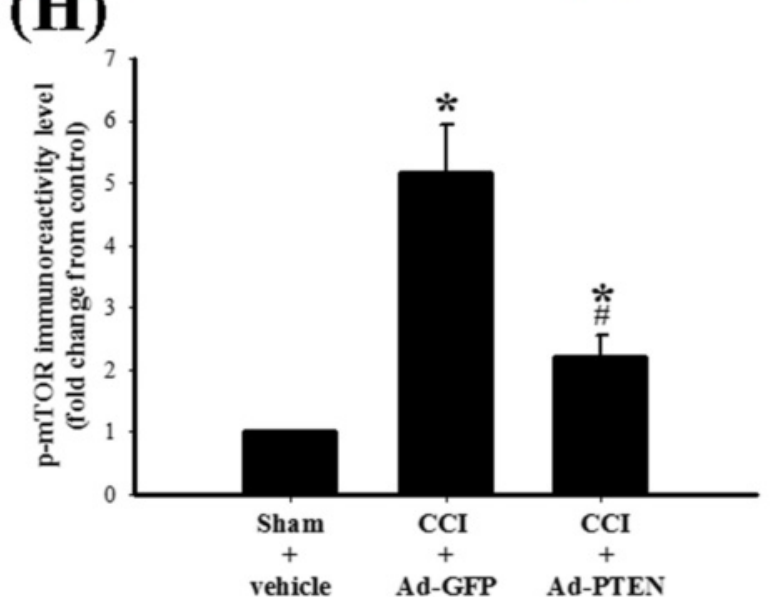

Figure 9 The inhibitive effect of i.t. Ad-PTEN on CCI-induced downregulation of PTEN and upregulation of phospho-mTOR. Spinal cord sections $(10 \mu \mathrm{m})$ obtained 14 days post-surgery from sham-operated plus i.t. vehicle (A, D), CCl plus i.t. Ad-GFP (B, E), and CCI plus i.t. Ad-PTEN (C, F) groups. Immunostaining images show cells labeled with PTEN (red) (A-C) and phospho-mTOR (red) (D-F) in the spinal cord. Quantification of PTEN (G) and phospho-mTOR (H) immunoreactivity in the ipsilateral dorsal horn of the lumbar spinal gray matter, and each bar in (G-H) represents the mean \pm SEM with six rats per group. Ad-PTEN (i.t.) significantly attenuated CCl-induced downregulation of spinal PTEN immunoreactivity and upregulation of spinal phospho-mTOR immunoreactivity. Scale bars: $200 \mu \mathrm{m}$ for all images (A-F). ${ }^{*} P<0.05$ compared with sham-operated plus i.t. vehicle group; ${ }^{\#} P<0.05$ compared with CCI plus i.t. Ad-GFP. Ad-GFP, adenovirus-mediated green fluorescent protein; Ad-PTEN, adenovirus-mediated phosphatase and tensin homolog deleted from chromosome 10; CCl, chronic constriction injury; p-mTOR, phosphorylated mammalian target of rapamycin.

the activation status of mTOR [59], a downstream inhibitory target of PTEN [10-12]. However, there is a lack of information regarding the changes in spinal phospho-
mTOR in CCI rats. Indeed, we found that upregulation of phospho-mTOR (Figure 1L) was associated with upregulation of phospho-PTEN (Figure 1J) after CCI 

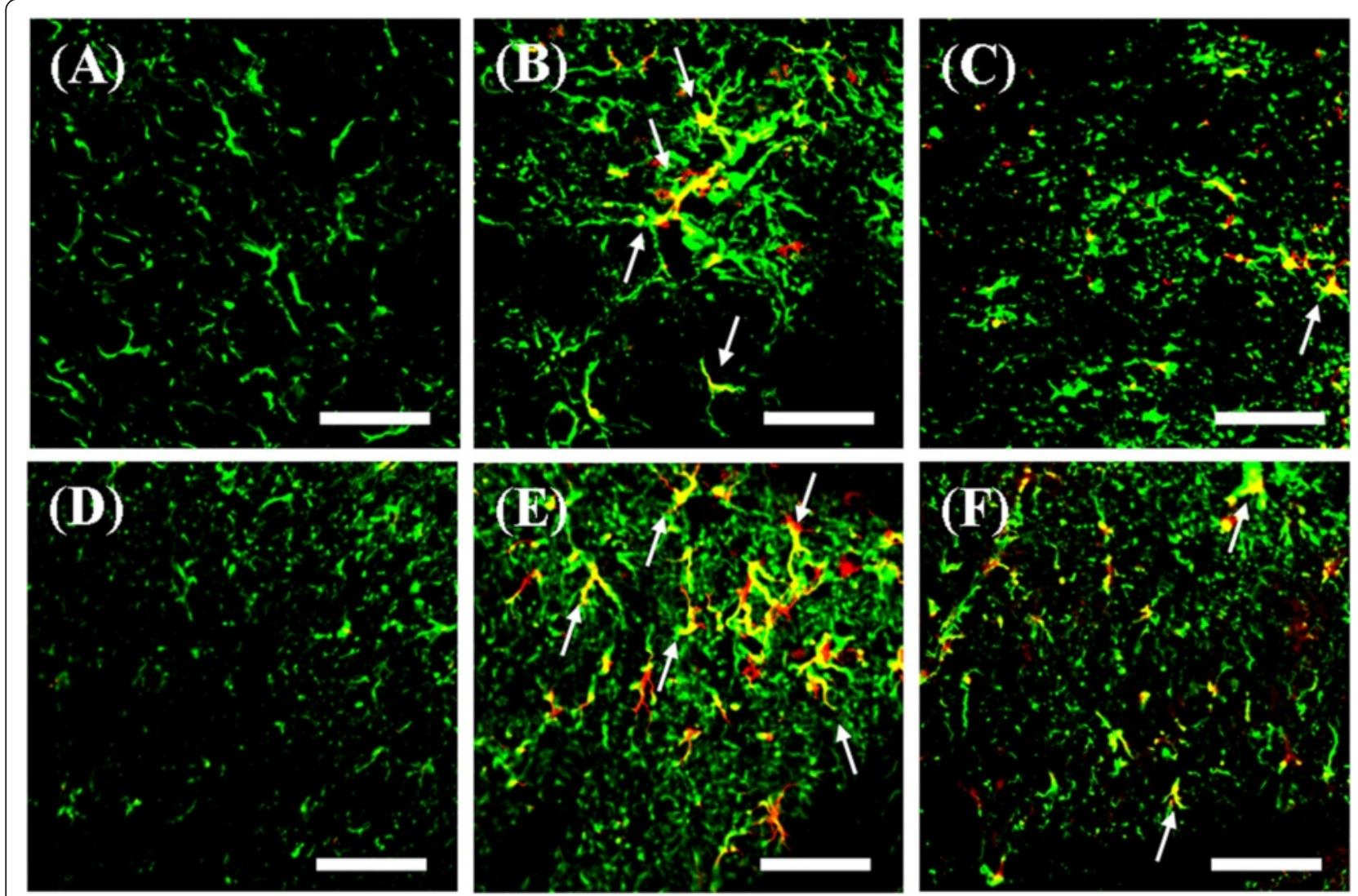

Figure 10 The effects of i.t. Ad-PTEN on CCI-induced upregulation of TNF- $a$ and phospho-mTOR in spinal astrocytes. Spinal cord sections $(10 \mu \mathrm{m})$ obtained 14 days post-surgery from sham-operated plus i.t. vehicle (A, D), CCl plus i.t. Ad-GFP (B, E), and CCI plus i.t. Ad-PTEN (C, F) groups. Confocal double-immunofluorescent staining of TNF-a (red; A-C) or phospho-mTOR (red; D-F) with GFAP (astrocyte specific marker; green) in the dorsal horn region of the lumbar spinal cord. The merged images (yellow; white arrow) indicate co-localization of TNF-a or phospho-mTOR with GFAP immunoreactive cells in the spinal cord. The confocal results showed that spinal TNF-a and phospho-mTOR were primarily co-localized with astrocytes in the CCI group, whereas i.t. Ad-PTEN significantly attenuated CCl-induced upregulation of spinal TNF-a and phospho-mTOR immunoreactivity in astrocytes. Scale bars are $50 \mu \mathrm{m}$ for all images.

surgery. After examining the changes in endogenous PTEN levels in neuropathic rats, we explored the potential effects of modulating spinal PTEN pathway on neuropathic pain. We observed that rats exhibited mechanical allodynia after i.t. injection of Ad-antisense PTEN (Figure 3B), which confirms that downregulation of spinal PTEN is important for nociception, and therefore, we suspect that spinal PTEN exerts beneficial effects against pain.

\section{The analgesic effects of spinal PTEN}

Clinical neuropathic pain syndromes are characterized by evoked pain (hyperalgesia and allodynia) or spontaneous pain [60]. We have confirmed these nociceptive behaviors in the rat $\mathrm{CCI}$ model, including thermal hyperalgesia (Figure 5A), mechanical and cold allodynia (Figure 5B,C), and weight-bearing deficits (spontaneous pain; Figure 5D). Ad-PTEN administered i.t. significantly prevented CCI-induced development of pain behaviors in neuropathic rats, including thermal hyperalgesia, mechanical allodynia, and cold allodynia accompanied with weight-bearing deficits (Figure 5). Central sensitization within the dorsal horn of the spinal cord could contribute to the hypersensitive pain behaviors commonly observed with neuropathic pain [61]. Activation of microglia and astrocytes could dominate spinal neuroinflammation $[62,63]$ and accelerate central sensitization as well as the subsequent development and maintenance of neuropathic pain $[20,21]$. Upregulation of OX-42 (microglial marker) and GFAP (astrocytic marker) immunoreactivity in the spinal dorsal horn are known indicators of elevated nociceptive states [23-25,64-69], which was further confirmed in the CCI rat model by our spinal immunohistofluorescence data (Figure 7). Many studies have demonstrated that inhibition of microglial and astrocytic activation exerts analgesic effects [19,23-25]. Although minocycline, a microglial inhibitor, can prevent neuropathic pain, it cannot reverse established 
neuropathic pain, thereby showing the important role of spinal microglia in the development phase but not in the maintenance phase of neuropathic pain $[25,70,71]$. However, it is still controversial whether spinal microglia contributes to the maintenance of nociception in neuropathy. As previously reported by Stuesse et al., OX-42 activity substantially peaked at around day 7 after nerve injury, and this increase was maintained for 35 days post-injury [68]. Several studies also suggest that microglial activation is probably important for the maintenance as well as the early development of neuropathic pain $[31,72,73]$. A series of studies have shown that astrocytic activation is implicated in the maintenance of neuropathic pain [64-67,69], indicating the key role of astrocytes in maintaining neuropathic pain $[74,75]$. Activated astrocytes are also reported to be important contributors of neuropathic pain development [76]. Our previous studies $[19,42,77]$ and our present findings also indicate that nociceptive behaviors accompany spinal microglial and astrocytic activation (Figure 7) in CCI rats. Moreover, our present findings also demonstrate that the upregulation of spinal PTEN by i.t. Ad-PTEN can significantly attenuate CCI-induced microglial and astrocytic activation in neuropathic rats (Figure 7).

\section{The majority of spinal cells affected by PTEN}

The expression and localization of PTEN in pain-related regions of the central nervous system are not completely understood. Our confocal double-immunostaining images of the lumbar spinal dorsal gray matter further confirmed that astrocytes are a major source of PTEN expression as compared to neurons and microglia in sham-operated rats (Figure 2). But certainly, the possibility that neurons and microglia could also be the source of PTEN expression cannot be excluded. This finding supports the hypothesis that the spinal astrocytic response plays a crucial role in neuropathic pain [19]. Ad-PTEN administered via i.t. injection significantly upregulated spinal PTEN immunoreactivity (Figure 4D). Previous studies have shown that the majority of spinal cells with adenovirus-mediated target gene overexpression were astrocytes $[78,79]$. Similar to the above studies, the present confocal results showed that spinal PTEN was primarily co-localized with astrocytes in the i.t. AdPTEN group (Figure 4E,F,G). Moreover, our present findings also showed that i.t. Ad-PTEN significantly attenuated CCI-induced astrocytic activation in neuropathic rats (Figure 7). This phenomenon was partially echoed by the finding that PTEN loss resulted in hypertrophy and increased proliferation of astrocytes in vivo [80], which are two characteristics of activated astrocytes [81]. Based on the above reports and the present results, we suggest that the prevention of neuropathic pain by i.t. Ad-PTEN may be mainly associated with its inhibitory effects on activated astrocytes, which play key roles in nociceptive hypersensitization. Previous studies have demonstrated that microglia could interact with astrocytes, causing downstream biochemical and behavioral responses to noxious stimuli [82-84]. Additionally, mediators released by activated microglia induce spinal astrocyte activation, and blocking these mediators attenuates astrocyte activation and pain [82-85]. Furthermore, mediators released by activated astrocytes, in turn, also activate spinal microglia in neuropathic or focal brain-injured rodents [86,87]. These findings from previous studies suggest that spinal microglia-astrocyte interactions could promote nociceptive responses (Figure 11A). Therefore, in this study, we hypothesized that the effect of the upregulation of spinal PTEN by i.t. Ad-PTEN on attenuating CCI-induced microglial activation may be secondary to the direct inhibition of activated astrocytes through suppressing the astrocytemicroglia interaction by reducing the release of astrocytic mediators (Figure 11B).

\section{Potential analgesic mechanisms of PTEN}

Previous studies have found that the activation of spinal mTOR is required for CCI-induced pain hypersensitivity [47]. Inhibition of the spinal mTOR pathway by i.t. rapamycin [13] or spinally administered rapamycin ester analogue CCI-779 [14] attenuates mechanical hyperalgesia in neuropathic rats. The above studies suggested that the anti-nociceptive effects produced by inhibition of the spinal mTOR pathway in neuropathic rats are consistent with a deleterious role for mTOR in neuropathic rats. In the present study, we observed upregulation of phosphomTOR following CCI surgery (Figure $1 \mathrm{~L}$ ). The findings of cancer research studies have indicated that PTEN is an upstream inhibitory mediator of mTOR, which also has been confirmed in the central nervous system (CNS) [10-12]. Our results indicated that i.t. Ad-PTEN significantly attenuated CCI-induced upregulation of spinal phospho-mTOR immunoreactivity (Figures 8 and 9). Moreover, similar to the systemic anti-inflammatory effects of intra-articular Ad-PTEN [17], i.t. Ad-PTEN also has central anti-inflammatory effects and can inhibit CCI-induced upregulation of spinal TNF- $\alpha$ (Figure 7). TNF- $\alpha$, a neuropathic pain-related cytokine [26], caused thermal hyperalgesia and mechanical allodynia in rats [27]. Moreover, neuropathic mechanical allodynia in rats was blocked by i.t. injection of a TNF-soluble receptor, a TNF antagonist [28]. In the present study, the confocal results from CCI rats showed that upregulated phospho-mTOR and TNF- $\alpha$ were localized predominantly to astrocytes, and this upregulation was inhibited by i.t. Ad-PTEN (Figure 10). Rapamycin, a specific inhibitor of mTOR, was reported to inhibit TNF- $\alpha$ release from human vascular smooth muscle cells in vitro [88]. Other studies have reported that rapamycin inhibition of mTOR in microglia [89] and 


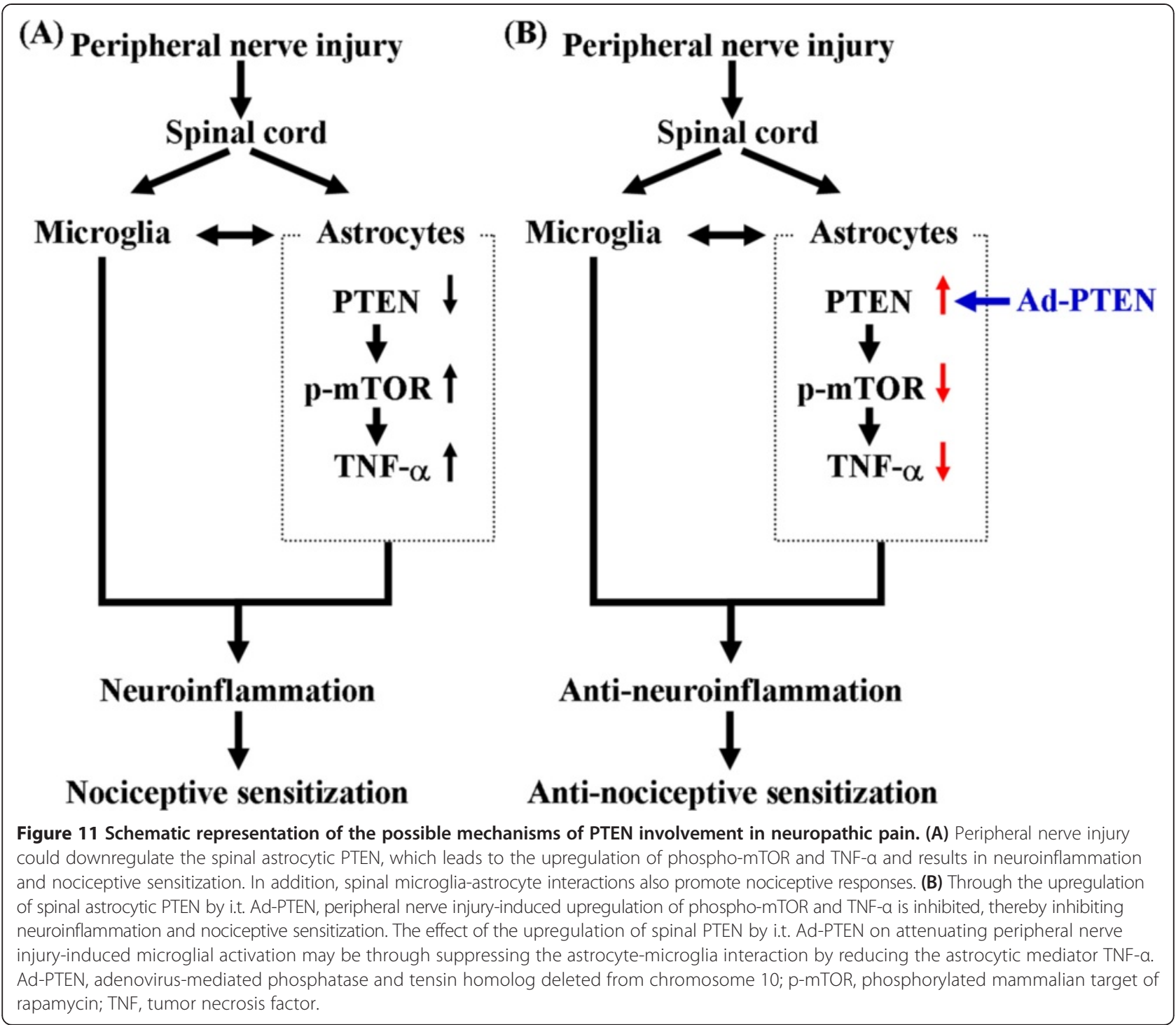

astrocytes [90] produces anti-inflammatory effects, which suggests that inhibiting mTOR can reduce neuroinflammation [91]. Thus, according to the previous and present findings, we conclude that one analgesic mechanism of PTEN may be inhibition of the astrocytic inflammatory response (upregulated TNF- $\alpha$ ) via inhibition of phosphomTOR (Figure 11B).

Advantages and future studies of PTEN for neuropathic pain We suspect that the upregulation of PTEN may be therapeutic for neuropathic pain, a neuroinflammationrelated disorder. Our present results demonstrate that PTEN exerts beneficial effects against neuropathic pain, which highlights three future research directions. First, it is important and valuable to explore the functions of PTEN through neuroscience research, especially neuroinflammation-related disorder mediated by activated astrocytes. However, a few in vitro [89] and in vivo [92] studies showed that inhibition of mTOR may modulate neurons or microglial activation. Therefore, the possibility that i.t. Ad-PTEN directly affects spinal neurons or microglia cannot be excluded. Second, Sims et al. [93] considered the adenovirus to be an efficient and safe vector for CNS gene delivery, although there are limitations to adenoviral-mediated PTEN gene therapy. The individuals treated with adenoviral vectors were previously reported to have experienced systemic reactions including fever, chill, and hypertension [48]. Although i.t. Ad-PTEN did not significantly affect the response to thermal hyperalgesia, mechanical allodynia, or cold allodynia tests on the contralateral hindpaw in CCI rats (Figure 6). Additionally, i.t. Ad-PTEN-treated 
normal rats and CCI rats did not exhibit any obvious abnormal external behavior (including locomotor function). The minimal effective dose and the duration of PTEN gene therapy still need to be determined in future research. Third, there is no known PTEN activator for neuropathic pain, and upregulation of PTEN may serve as an in vitro index to screen compounds that may be potential neuropathic pain therapies.

\section{Conclusions}

This study is a novel step toward understanding the benefits of PTEN on neuropathic pain and provides a foundation for future studies utilizing upregulation of PTEN in combinational therapies for neuropathic pain.

\section{Competing interests}

The authors declare that they have no competing interests.

\section{Authors' contributions}

SYH, CSS, MHT, and ZHW conceived and designed the experiments. SYH, CSS, CHC, CWF, and HCH performed the experiments. SYH, CSS, WFC, SNY, NFC, and HMDW analyzed the data. PRL, SCC, and THC contributed to the preparation of adenovirus vectors. SYH, CSS, MHT, and ZHW contributed to the writing of the manuscript. All authors have read and approved the final version of the manuscript.

\section{Acknowledgements}

The study was supported by research grants from the Ministry of Science and Technology, Taiwan (102-2325-B-110-002, 101-2314-B-182A-023-MY2, 103-2325-B-110 -001, and 103-2314-B-075 -031) and the Taipei Veterans General Hospital, Taiwan (V102C-198). We also thank the projects of Center for Stem Cell Research, Kaohsiung Medical University, Kaohsiung, Taiwan, KMU-TP103G00 and KMU-TP103G02-05.

\section{Author details}

1 Department of Marine Biotechnology and Resources, National Sun Yat-sen University, No. 70, Lienhai Road, Kaohsiung 80424, Taiwan. ${ }^{2}$ Center for Neuroscience, National Sun Yat-sen University, No. 70, Lienhai Road, Kaohsiung 80424, Taiwan. ${ }^{3}$ Department of Anesthesiology, Taipei Veterans General Hospital, No. 201, Section 2, Shipai Road, Taipei 11217, Taiwan. ${ }^{4}$ School of Medicine, National Yang-Ming University, No. 155, Section 2, Linong Street, Taipei 11221, Taiwan. ${ }^{5}$ Department of Neurosurgery, Kaohsiung Chang Gung Memorial Hospital and Chang Gung University College of Medicine, No. 123, DAPI Road, Kaohsiung 83301, Taiwan. ${ }^{6}$ Department of Neurosurgery, Xiamen Chang Gung Memorial Hospital, No. 123, Xiafei Road, Fujian 361026, China. ${ }^{7}$ Doctoral Degree Program in Marine Biotechnology, National Sun Yat-sen University and Academia Sinica, No. 70, Lienhai Road, Kaohsiung 80424, Taiwan. ${ }^{8}$ School of Medicine, College of Medicine and Department of Pediatrics, E-DA Hospital, I-Shou University, No. 1, Yida Road, Kaohsiung 82445, Taiwan. ${ }^{9}$ Division of Neurosurgery, Department of Surgery, Kaohsiung Armed Forces General Hospital, No. 2, Zhongzheng 1st Road, Kaohsiung 80284, Taiwan. ${ }^{10}$ Institute of Biomedical Sciences, National Sun Yat-sen University, \#70 Lienhai Road, Kaohsiung 80424, Taiwan. ${ }^{11}$ Department of Fragrance and Cosmetic Science, Kaohsiung Medical University, No. 100, Shiquan 1st Road, Kaohsiung 80708, Taiwan. ${ }^{12}$ Graduate Institute of Natural Products, Kaohsiung Medical University, No. 100, Shiquan 1st Road, Kaohsiung 80708, Taiwan. ${ }^{13}$ Center for Stem Cell Research, Kaohsiung Medical University, No. 100, Shiquan 1st Road, Kaohsiung 80708, Taiwan. ${ }^{14}$ Department of Biological Sciences, National Sun Yat-sen University, No. 70, Lienhai Road, Kaohsiung 80424, Taiwan. ${ }^{15}$ Marine Biomedical Laboratory and Center for Translational Biopharmaceuticals, Department of Marine Biotechnology and Resources, National Sun Yat-sen University, No. 70, Lienhai Road, Kaohsiung 80424, Taiwan.

Received: 22 October 2014 Accepted: 7 March 2015

Published online: 26 March 2015

\section{References}

1. Sprintz M, Tasciotti E, Allegri M, Grattoni A, Driver LC, Ferrari M. Nanomedicine: ushering in a new era of pain management. Eur J Pain Suppl. 2011;5:317-22.

2. Melnikova I. Pain market. Nat Rev Drug Discov. 2010;9:589-90.

3. Breivik H, Collett B, Ventafridda V, Cohen R, Gallacher D. Survey of chronic pain in Europe: prevalence, impact on daily life, and treatment. Eur J Pain. 2006;10:287-333

4. Bouhassira D, Lantéri-Minet M, Attal N, Laurent B, Touboul C. Prevalence of chronic pain with neuropathic characteristics in the general population. Pain. 2008;136:380-7.

5. Finnerup NB, Sindrup SH, Jensen TS. The evidence for pharmacological treatment of neuropathic pain. Pain. 2010;150:573-81.

6. Zhang P, Chen JH, Guo XL. New insights into PTEN regulation mechanisms and its potential function in targeted therapies. Biomed Pharmacother. 2012;66:485-90

7. Song MS, Salmena L, Pandolfi PP. The functions and regulation of the PTEN tumour suppressor. Nat Rev Mol Cell Biol. 2012;13:283-96.

8. Chetram MA, Hinton CV. PTEN regulation of ERK1/2 signaling in cancer. J Recept Signal Transduct Res. 2012;32:190-5.

9. Goebbels S, Oltrogge JH, Wolfer S, Wieser GL, Nientiedt T, Pieper A, et al. Genetic disruption of Pten in a novel mouse model of tomaculous neuropathy. EMBO Mol Med. 2012;4:486-99.

10. Kim WY, Snider WD. Neuroscience. Overcoming inhibitions. Science. 2008;322:869-72

11. Park KK, Liu K, Hu Y, Smith PD, Wang C, Cai B, et al. Promoting axon regeneration in the adult CNS by modulation of the PTEN/mTOR pathway. Science. 2008:322:963-6.

12. Shi GD, OuYang YP, Shi JG, Liu Y, Yuan W, Jia LS. PTEN deletion prevents ischemic brain injury by activating the mTOR signaling pathway. Biochem Biophys Res Commun. 2011;404:941-5.

13. Geranton SM, Jimenez-Diaz L, Torsney C, Tochiki KK, Stuart SA, Leith JL, et al. A rapamycin-sensitive signaling pathway is essential for the full expression of persistent pain states. J Neurosci. 2009;29:15017-27.

14. Asante $\mathrm{CO}$, Wallace VC, Dickenson AH. Mammalian target of rapamycin signaling in the spinal cord is required for neuronal plasticity and behavioral hypersensitivity associated with neuropathy in the rat. J Pain. 2010;11:1356-67.

15. Kung ML, Tsai HE, Hu TH, Kuo HM, Liu LF, Chen SC, et al. Hepatoma-derived growth factor stimulates podosome rosettes formation in $\mathrm{NIH} / 3$ T3 cells through the activation of phosphatidylinositol 3-kinase/Akt pathway. Biochem Biophys Res Commun. 2012;425:169-76.

16. Kuo HM, Lin CY, Lam HC, Lin PR, Chan HH, Tseng JC, et al. PTEN overexpression attenuates angiogenic processes of endothelial cells by blockade of endothelin-1/endothelin B receptor signaling. Atherosclerosis. 2012;221:341-9.

17. Wang CR, Shiau AL, Chen SY, Lin LL, Tai MH, Shieh GS, et al. Amelioration of collagen-induced arthritis in rats by adenovirus-mediated PTEN gene transfer. Arthritis Rheum. 2008:58:1650-6.

18. Huang SY, Chen NF, Chen WF, Hung HC, Lee HP, Lin YY, et al. Sinularin from indigenous soft coral attenuates nociceptive responses and spinal neuroinflammation in carrageenan-induced inflammatory rat model. Mar Drugs. 2012;10:1899-919.

19. Lin YC, Huang SY, Jean YH, Chen WF, Sung CS, Kao ES, et al. Intrathecal lemnalol, a natural marine compound obtained from Formosan soft coral, attenuates nociceptive responses and the activity of spinal glial cells in neuropathic rats. Behav Pharmacol. 2011;22:739-50.

20. Milligan ED, Watkins LR. Pathological and protective roles of glia in chronic pain. Nat Rev Neurosci. 2009;10:23-36.

21. Marchand F, Perretti M, McMahon SB. Role of the immune system in chronic pain. Nat Rev Neurosci. 2005;6:521-32.

22. Chen NF, Huang SY, Chen WF, Chen CH, Lu CH, Chen CL, et al. TGF- $\beta 1$ attenuates spinal neuroinflammation and the excitatory amino acid system in rats with neuropathic pain. J Pain. 2013:14:1671-85.

23. Jean YH, Chen WF, Sung CS, Duh CY, Huang SY, Lin CS, et al. Capnellene, a natural marine compound derived from soft coral, attenuates chronic constriction injury-induced neuropathic pain in rats. Br J Pharmacol. 2009;158:713-25.

24. Sweitzer SM, Schubert P, DeLeo JA. Propentofylline, a glial modulating agent, exhibits antiallodynic properties in a rat model of neuropathic pain. J Pharmacol Exp Ther. 2001;297:1210-7.

25. Ledeboer A, Sloane EM, Milligan ED, Frank MG, Mahony JH, Maier SF, et al. Minocycline attenuates mechanical allodynia and proinflammatory cytokine expression in rat models of pain facilitation. Pain. 2005;115:71-83. 
26. Leung L, Cahill CM. TNF-alpha and neuropathic pain - a review. J Neuroinflammation. 2010;7:27.

27. Youn DH, Wang H, Jeong SJ. Exogenous tumor necrosis factor-alpha rapidly alters synaptic and sensory transmission in the adult rat spinal cord dorsal horn. J Neurosci Res. 2008;86:2867-75.

28. Milligan ED, Twining C, Chacur M, Biedenkapp J, O'Connor K, Poole S, et al. Spinal glia and proinflammatory cytokines mediate mirror-image neuropathic pain in rats. J Neurosci. 2003;23:1026-40

29. Bennett GJ, Xie YK. A peripheral mononeuropathy in rat that produces disorders of pain sensation like those seen in man. Pain. 1988;33:87-107.

30. Yaksh TL, Rudy TA. Chronic catheterization of the spinal subarachnoid space. Physiol Behav. 1976;17:1031-6.

31. Hains BC, Waxman SG. Activated microglia contribute to the maintenance of chronic pain after spinal cord injury. J Neurosci. 2006;26:4308-17.

32. Basso DM, Beattie MS, Bresnahan JC. A sensitive and reliable locomotor rating scale for open field testing in rats. J Neurotrauma. 1995:12:1-21.

33. Hargreaves $K$, Dubner $R$, Brown F, Flores C, Joris J. A new and sensitive method for measuring thermal nociception in cutaneous hyperalgesia. Pain. 1988;32:77-88.

34. Jean YH, Chen WF, Duh CY, Huang SY, Hsu CH, Lin CS, et al. Inducible nitric oxide synthase and cyclooxygenase-2 participate in anti-inflammatory and analgesic effects of the natural marine compound lemnalol from Formosan soft coral Lemnalia cervicorni. Eur J Pharmacol. 2008:578:323-31.

35. Chaplan SR, Bach FW, Pogrel JW, Chung JM, Yaksh TL. Quantitative assessment of tactile allodynia in the rat paw. J Neurosci Methods. 1994:53:55-63.

36. Bardin L, Malfetes N, Newman-Tancredi A, Depoortere R. Chronic restraint stress induces mechanical and cold allodynia, and enhances inflammatory pain in rat: relevance to human stress-associated painful pathologies. Behav Brain Res. 2009;205:360-6.

37. Flatters SJ, Bennett GJ. Ethosuximide reverses paclitaxel- and vincristine-induced painful peripheral neuropathy. Pain. 2004;109:150-61.

38. Wen ZH, Tang CC, Chang YC, Huang SY, Hsieh SP, Lee $\mathrm{CH}$, et al. Glucosamine sulfate reduces experimental osteoarthritis and nociception in rats: association with changes of mitogen-activated protein kinase in chondrocytes. Osteoarthritis Cartilage. 2010;18:1192-202.

39. Kobayashi K, Imaizumi R, Sumichika H, Tanaka H, Goda M, Fukunari A, et al. Sodium iodoacetate-induced experimental osteoarthritis and associated pain model in rats. J Vet Med Sci. 2003;65:1195-9.

40. Sung B, Lim G, Mao J. Altered expression and uptake activity of spinal glutamate transporters after nerve injury contribute to the pathogenesis of neuropathic pain in rats. J Neurosci. 2003;23:2899-910.

41. Raposo D, Morgado C, Pereira-Terra P, Tavares I. Nociceptive spinal cord neurons of laminae I-III exhibit oxidative stress damage during diabetic neuropathy which is prevented by early antioxidant treatment with epigallocatechin-gallate (EGCG). Brain Res Bull. 2015;110:68-75.

42. Chen NF, Huang SY, Lu CH, Chen CL, Feng CW, Chen $\mathrm{CH}$, et al. Flexibilide obtained from cultured soft coral has anti-neuroinflammatory and analgesic effects through the upregulation of spinal transforming growth factor-beta1 in neuropathic rats. Mar Drugs. 2014;12:3792-817.

43. Stokes JA, Cheung J, Eddinger K, Corr M, Yaksh TL. Toll-like receptor signaling adapter proteins govern spread of neuropathic pain and recovery following nerve injury in male mice. J Neuroinflammation. 2013;10:148.

44. Kawasaki $Y, X u Z Z$, Wang $X$, Park JY, Zhuang ZY, Tan PH, et al. Distinct roles of matrix metalloproteases in the early- and late-phase development of neuropathic pain. Nat Med. 2008;14:331-6.

45. Chen WF, Sung CS, Jean YH, Su TM, Wang HC, Ho JT, et al. Suppressive effects of intrathecal granulocyte colony-stimulating factor on excessive release of excitatory amino acids in the spinal cerebrospinal fluid of rats with cord ischemia: role of glutamate transporters. Neuroscience. 2010;165:1217-32.

46. Lowry OH, Rosebrough NJ, Farr AL, Randall RJ. Protein measurement with the Folin phenol reagent. J Biol Chem. 1951;193:265-75.

47. Zhang W, Sun XF, Bo JH, Zhang J, Liu XJ, Wu LP, et al. Activation of mTOR in the spinal cord is required for pain hypersensitivity induced by chronic constriction injury in mice. Pharmacol Biochem Behav. 2013;111:64-70.

48. Shi JY, Liu GS, Liu LF, Kuo SM, Ton CH, Wen ZH, et al. Glial cell line-derived neurotrophic factor gene transfer exerts protective effect on axons in sciatic nerve following constriction-induced peripheral nerve injury. Hum Gene Ther. 2011;22:721-31.

49. Austin PJ, Wu A, Moalem-Taylor G. Chronic constriction of the sciatic nerve and pain hypersensitivity testing in rats. J Vis Exp. 2012.
50. Zhang L, Sun T, Yu E, Yu L, Luo J, Li H, et al. TNF-alpha expression, not iNOS expression, is correlated with NF-kappaB activation in the spinal cord of rats following peripheral nerve injury. Afr J Biotechnol. 2011;10:6372-80.

51. Park KK, Liu K, Hu Y, Kanter JL, He Z. PTEN/mTOR and axon regeneration. Exp Neurol. 2010;223:45-50.

52. Liu K, Lu Y, Lee JK, Samara R, Willenberg R, Sears-Kraxberger I, et al. PTEN deletion enhances the regenerative ability of adult corticospinal neurons. Nat Neurosci. 2010;13:1075-81.

53. Sonoda Y, Mukai H, Matsuo K, Takahashi M, Ono Y, Maeda K, et al. Accumulation of tumor-suppressor PTEN in Alzheimer neurofibrillary tangles. Neurosci Lett. 2010;471:20-4.

54. Diaz-Ruiz O, Zapata A, Shan L, Zhang Y, Tomac AC, Malik N, et al. Selective deletion of PTEN in dopamine neurons leads to trophic effects and adaptation of striatal medium spiny projecting neurons. PLoS One. 2009;4: e7027.

55. Liu G, Detloff MR, Miller KN, Santi L, Houle JD. Exercise modulates microRNAs that affect the PTEN/mTOR pathway in rats after spinal cord injury. Exp Neurol. 2012;233:447-56

56. Walker CL, Walker MJ, Liu NK, Risberg EC, Gao X, Chen J, et al. Systemic bisperoxovanadium activates Akt/mTOR, reduces autophagy, and enhances recovery following cervical spinal cord injury. PLoS One. 2012;7:e30012.

57. Liu G, Keeler BE, Zhukareva V, Houle JD. Cycling exercise affects the expression of apoptosis-associated microRNAs after spinal cord injury in rats. Exp Neurol. 2010;226:200-6.

58. Oinuma I, Ito $Y$, Katoh H, Negishi M. Semaphorin 4D/Plexin-B1 stimulates PTEN activity through R-Ras GTPase-activating protein activity, inducing growth cone collapse in hippocampal neurons. J Biol Chem. 2010:285:28200-9.

59. Chiang GG, Abraham RT. Phosphorylation of mammalian target of rapamycin (mTOR) at Ser-2448 is mediated by p70S6 kinase. J Biol Chem. 2005;280:25485-90

60. Baron R. Neuropathic pain: a clinical perspective. Handb Exp Pharmacol. 2009;194:3-30. doi:10.1007/978-3-540-79090-7_1.

61. Costigan M, Scholz J, Woolf CJ. Neuropathic pain: a maladaptive response of the nervous system to damage. Annu Rev Neurosci. 2009;32:1-32.

62. Myers RR, Campana WM, Shubayev VI. The role of neuroinflammation in neuropathic pain: mechanisms and therapeutic targets. Drug Discov Today. 2006;11:8-20.

63. Streit WJ, Mrak RE, Griffin WS. Microglia and neuroinflammation: a pathological perspective. J Neuroinflammation. 2004;1:14.

64. Garrison CJ, Dougherty PM, Kajander KC, Carlton SM. Staining of glia fibrillary acidic protein (GFAP) in lumbar spinal cord increases following a sciatic nerve constriction injury. Brain Res. 1991;565:1-7.

65. Colburn RW, DeLeo JA, Rickman AJ, Yeager MP, Kwon P, Hickey WF. Dissociation of microglial activation and neuropathic pain behaviors following peripheral nerve injury in the rat. J Neuroimmunol. 1997;79:163-75.

66. Colburn RW, Rickman AJ, DeLeo JA. The effect of site and type of nerve injury on spinal glial activation and neuropathic pain behavior. Exp Neurol. 1999;157:289-304

67. Coyle DE. Partial peripheral nerve injury leads to activation of astroglia and microglia which parallels the development of allodynic behavior. Glia. 1998;23:75-83.

68. Stuesse SL, Cruce WL, Lovell JA, McBurney DL, Crisp T. Microglial proliferation in the spinal cord of aged rats with a sciatic nerve injury. Neurosci Lett. 2000;287:121-4.

69. Stuesse SL, Crisp T, McBurney DL, Schechter JB, Lovell JA, Cruce WL. Neuropathic pain in aged rats: behavioral responses and astrocytic activation. Exp Brain Res. 2001;137:219-27.

70. Raghavendra V, Tanga F, DeLeo JA. Inhibition of microglial activation attenuates the development but not existing hypersensitivity in a rat model of neuropathy. J Pharmacol Exp Ther. 2003;306:624-30.

71. Padi SS, Kulkarni SK. Minocycline prevents the development of neuropathic pain, but not acute pain: possible anti-inflammatory and antioxidant mechanisms. Eur J Pharmacol. 2008:601:79-87.

72. Ji RR, Suter MR. p38 MAPK, microglial signaling, and neuropathic pain. Mol Pain. 2007;3:33.

73. Cao L, Palmer CD, Malon JT, De Leo JA. Critical role of microglial CD40 in the maintenance of mechanical hypersensitivity in a murine model of neuropathic pain. Eur J Immunol. 2009;39:3562-9.

74. Zhuang ZY, Gerner P, Woolf CJ, Ji RR. ERK is sequentially activated in neurons, microglia, and astrocytes by spinal nerve ligation and 
contributes to mechanical allodynia in this neuropathic pain model. Pain. 2005;114:149-59.

75. Zhuang ZY, Wen YR, Zhang DR, Borsello T, Bonny C, Strichartz GR, et al. A peptide c-Jun N-terminal kinase (JNK) inhibitor blocks mechanical allodynia after spinal nerve ligation: respective roles of JNK activation in primary sensory neurons and spinal astrocytes for neuropathic pain development and maintenance. J Neurosci. 2006:26:3551-60.

76. Moon JY, Roh DH, Yoon SY, Choi SR, Kwon SG, Choi HS, et al. Sigma1 receptors activate astrocytes via p38 MAPK phosphorylation leading to the development of mechanical allodynia in a mouse model of neuropathic pain. Br J Pharmacol. 2014;171:5881-97.

77. Chen WF, Huang SY, Liao CY, Sung CS, Chen JY, Wen ZH. The use of the antimicrobial peptide piscidin (PCD)-1 as a novel anti-nociceptive agent. Biomaterials. 2015;53:1-11.

78. Maeda S, Kawamoto A, Yatani Y, Shirakawa H, Nakagawa T, Kaneko S. Gene transfer of GLT-1, a glial glutamate transporter, into the spinal cord by recombinant adenovirus attenuates inflammatory and neuropathic pain in rats. Mol Pain. 2008;4:65.

79. Romero MI, Rangappa N, Li L, Lightfoot E, Garry MG, Smith GM. Extensive sprouting of sensory afferents and hyperalgesia induced by conditional expression of nerve growth factor in the adult spinal cord. J Neurosci. 2000;20:4435-45.

80. Fraser MM, Zhu X, Kwon CH, Uhlmann EJ, Gutmann DH, Baker SJ. Pten loss causes hypertrophy and increased proliferation of astrocytes in vivo. Cancer Res. 2004;64:7773-9.

81. Ferraguti F, Corti C, Valerio E, Mion S, Xuereb J. Activated astrocytes in areas of kainate-induced neuronal injury upregulate the expression of the metabotropic glutamate receptors 2/3 and 5. Exp Brain Res. 2001:137:1-11.

82. Raghavendra V, Tanga FY, DeLeo JA. Complete Freunds adjuvant-induced peripheral inflammation evokes glial activation and proinflammatory cytokine expression in the CNS. Eur J Neurosci. 2004;20:467-73.

83. Watkins LR, Martin D, Ulrich P, Tracey KJ, Maier SF. Evidence for the involvement of spinal cord glia in subcutaneous formalin induced hyperalgesia in the rat. Pain. 1997;71:225-35

84. Sung CS, Cherng CH, Wen ZH, Chang WK, Huang SY, Lin SL, et al. Minocycline and fluorocitrate suppress spinal nociceptive signaling in intrathecal IL-1 beta-induced thermal hyperalgesic rats. Glia. 2012;60:2004-17.

85. Miyoshi K, Obata K, Kondo T, Okamura H, Noguchi K. Interleukin-18-mediated microglia/astrocyte interaction in the spinal cord enhances neuropathic pain processing after nerve injury. J Neurosci. 2008;28:12775-87.

86. Davalos D, Grutzendler J, Yang G, Kim JV, Zuo Y, Jung S, et al. ATP mediates rapid microglial response to local brain injury in vivo. Nat Neurosci. 2005;8:752-8.

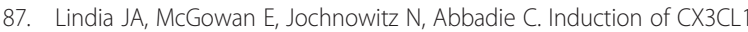
expression in astrocytes and CX3CR1 in microglia in the spinal cord of a rat model of neuropathic pain. J Pain. 2005;6:434-8.

88. Adkins JR, Castresana MR, Wang Z, Newman WH. Rapamycin inhibits release of tumor necrosis factor-alpha from human vascular smooth muscle cells. Am Surg. 2004;70:384-7. discussion 387-388.

89. Dello Russo C, Lisi L, Tringali G, Navarra P. Involvement of mTOR kinase in cytokine-dependent microglial activation and cell proliferation. Biochem Pharmacol. 2009;78:1242-51.

90. Lisi L, Navarra P, Feinstein D, Dello RC. The mTOR kinase inhibitor rapamycin decreases iNOS mRNA stability in astrocytes. J Neuroinflammation. 2011:8:1.

91. Dello Russo C, Lisi L, Feinstein DL, Navarra P. mTOR kinase, a key player in the regulation of glial functions: Relevance for the therapy of multiple sclerosis. Glia. 2013;61:301-11.

92. Norsted Gregory E, Codeluppi S, Gregory JA, Steinauer J, Svensson Cl. Mammalian target of rapamycin in spinal cord neurons mediates hypersensitivity induced by peripheral inflammation. Neuroscience. 2010;169:1392-402.

93. Sims K, Ahmed Z, Gonzalez AM, Read ML, Cooper-Charles L, Berry M, et al. Targeting adenoviral transgene expression to neurons. Mol Cell Neurosci. 2008:39:411-7.

\section{Submit your next manuscript to BioMed Central and take full advantage of:}

- Convenient online submission

- Thorough peer review

- No space constraints or color figure charges

- Immediate publication on acceptance

- Inclusion in PubMed, CAS, Scopus and Google Scholar

- Research which is freely available for redistribution

Submit your manuscript at www.biomedcentral.com/submit 